\title{
Integrative Genomic Analyses of 1,145 Patient Samples Reveal New Biomarkers in Esophageal Squamous Cell Carcinoma
}

\author{
Binbin Zou ${ }^{1,2}$, Dinghe Guo ${ }^{1,2}$, Pengzhou Kong ${ }^{1,2}$, Yanqiang Wang ${ }^{1,2}$, Xiaolong Cheng ${ }^{1,2 *}$ and \\ Yongping Cui ${ }^{1,2,3 *}$
}

${ }^{1}$ Key Laboratory of Cellular Physiology of the Ministry of Education, Shanxi Medical University, Taiyuan, China, ${ }^{2}$ Department of Pathology, Shanxi Medical University, Taiyuan, China, ${ }^{3}$ Shenzhen Peking University-Hong Kong University of Science and Technology (PKU-HKUST) Medical Center, Peking University Shenzhen Hospital, Shenzhen, China

OPEN ACCESS

Edited by:

Xin Wang,

The Chinese University of Hong Kong, Hong Kong SAR, China

Reviewed by:

Feng Gao,

The Sixth Affiliated Hospital of Sun

Yat-sen University, China

Hao Huang,

City University of Hong Kong, Hong

Kong SAR, China

*Correspondence:

Xiaolong Cheng

chengx@@sxmu.edu.cn

Yongping $\mathrm{Cu}$

cuiyp@sxmu.edu.cn

Specialty section:

This article was submitted to

Molecular Diagnostics and

Therapeutics,

a section of the journa

Frontiers in Molecular Biosciences

Received: 11 October 2021 Accepted: 02 December 2021

Published: 21 January 2022

Citation:

Zou B, Guo D, Kong $P$, Wang $Y$, Cheng $X$ and Cui $Y$ (2022) Integrative

Genomic Analyses of 1,145 Patient

Samples Reveal New Biomarkers in

Esophageal Squamous

Cell Carcinoma.

Front. Mol. Biosci. 8:792779.

doi: 10.3389/fmolb.2021.792779
Due to the lack of effective diagnostic markers and therapeutic targets, esophageal squamous cell carcinoma (ESCC) shows a poor 5 years survival rate of less than $30 \%$. To explore the potential therapeutic targets of ESCC, we integrated and reanalyzed the mutation data of WGS (whole genome sequencing) or WES (whole exome sequencing) from a total of 1,145 samples in 7 large ESCC cohorts, including 270 ESCC gene expression data. Two new mutation signatures and 20 driver genes were identified in our study. Among them, AP3S1, MUC16, and RPS15 were reported for the first time. We also discovered that the KMT2D was associated with the multiple clinical characteristics of ESCC, and KMT2D knockdown cells showed enhanced cell migration and cell invasion. Furthermore, a few neoantigens were shared between ESCC patients. For ESCC, compared to TMB, neoantigen might be treated as a better immunotherapy biomarker. Our research expands the understanding of ESCC mutations and helps the identification of ESCC biomarkers, especially for immunotherapy biomarkers.

Keywords: esophageal squamous cell carcinoma, mutation, neoantigen, immunotherapy, KMT2D, bioinformactics

\section{INTRODUCTION}

Esophageal cancer (EC) is the fourth most common cancer in China, with 375,000 annual deaths, $90 \%$ of which are esophageal squamous cell carcinoma (ESCC) (Chen et al., 2016). Due to the lack of effective diagnostic markers, most of ESCC patients are diagnosed at the advanced stages and are not suitable for surgical treatment. Due to the limited treatment methods, the 5 years survival rate of ESCC is less than 30\% (Younes et al., 2002; Koshy et al., 2004). Therefore, it is imperative to explore new biomarkers and novel targets for ESCC diagnosis, prognosis, and clinical treatment.

In recent years, series of whole genome sequencing (WGS) and whole exome sequencing (WES) studies had been performed in ESCC (Lin et al., 2014; Song et al., 2014; Cao et al., 2015; Zhang et al., 2015; Hu et al., 2016; Qin et al., 2016; Sawada et al., 2016; Chang et al., 2017; Chen et al., 2017; Dai et al., 2017; Du et al., 2017; Liu et al., 2017; Nature, 2017; Dai et al., 2018; Yan et al., 2019; Yu et al., 2019; Cui et al., 2020; Dutta et al., 2020; He et al., 2020; Liu et al., 2020; Mangalaparthi et al., 2020). As a result of that, several ESCC driver genes had been identified. However, limitations in sample size and the algorithms used in driver gene identification still exist. More than half of the 
driver genes were identified in only one cohort, and few biomarkers were identified as especial immunotherapyrelated biomarkers. At the same time, because of the lack of therapeutic targets for ESCC, targeted therapy treatment has not been improved significantly.

Here, we performed an integrative analysis using multiple datasets from 7 published cohorts, 270 ESCC gene expression data to explore potential biomarker especially immunotherapy biomarkers of ESCC. We found that the integration of data with a larger sample size is helpful in not only discovering new mutation signatures and new driver genes, but also identifying more potential biomarkers, especially low-frequency mutation genes.

\section{METHODS}

\section{Data Collection and Analysis}

ESCC mutation data: The DNA mutation data was downloaded from the results of articles of 7 cohorts (Lin et al., 2014; Song et al., 2014; Zhang et al., 2015; Sawada et al., 2016; Chang et al., 2017; Nature, 2017; Cui et al., 2020), which provided whole mutation data, whole clinical information of sample and the sample number was bigger. All mutation results were uniformly converted into MAF format (https:/docs.gdc.cancer.gov/Data/ File_Formats/MAF_Format, Supplementary Table S2, Supplementary Table S3 was the clinical information of samples). ESCC gene expression data: 1) TCGA-ESCA gene expression data download from UCSC xena, different expression genes (DEGs) were identified by $t$ test. 2) GSE53625 chip data download from NCBI GEO. Use blast alignment for each probe of the GSE53625 chip data against the hg19 reference genome. $100 \%$ alignment was required, and mismatches and indels were not allowed. For multiple probes aligned to the same gene, the expression value of the gene was the average of all probes. There were a total of 270 ESCC gene expression data.

\section{Mutation Signature Analysis}

We used sigflow to analyze the mutation signature of the exon regions of all samples in SBS, DBS, ID types, and we also analyzed the whole genome of the 508 samples in SBS, DBS, ID types.

\section{TMB and Neoantigen Analysis}

TMB calculation: total TMB $(\mathrm{aTMB})=(\mathrm{SNV}+$ indel $) / 30 \mathrm{M}$, $\mathrm{fTMB}=($ exclude nonsynonymous $\mathrm{SNV}+$ indel $) / 30 \mathrm{M}$.

HLA allele typing: Use optitype to identify HLA-A, HLA-B, HLA-C alleles on the 90 WES samples.

Neoantigen identified: First used maf2vcf (https://github.com/ mskcc/vcf2maf) to convert the MAF to VCF, then used vep (McLaren et al., 2016) to annotate VCF referred to pvactools manual, next used pvactools to perform neoantigen analysis on samples. The BindLevel of the peptide with SB or WB as candidate neoantigen.

Tumor neoantigen burden (TNB): Count the number of all candidate neoantigen in the sample.
Tumor neoantigen score (TNS): The neoantigen score was the sum of the scores of all mutant peptides of the gene (Gscore).

$$
\text { Gsore }=\sum_{1}^{n} \frac{A \text { Ascore }}{M}, \text { AAscore }=\left\{\begin{array}{c}
\log _{2}(W / M) \\
15
\end{array}\right.
$$

Where Gscore represented the neoantigen score of the gene, W represented the binding score of wild-type peptide with the HLA allele, $M$ represented the binding score of the mutant peptide with the HLA allele, and AAscore represented the ratio of wild-type peptide binding score to mutant peptide binding score, if the mutant peptides did not have a corresponding wild-type peptide, such as mutant peptides produced by frameshift caused by indel, the ratio was 15 .

\section{Driver Gene Analysis}

MutSigCV, driverml, oncodrivefml, and oncodriveclutl were used to identify driver genes based on coding region mutation of 1145 ESCC patients. For driverml, the $p<0.01$ was used as the threshold, and for MutSigCV, oncodrivefml, oncodriveclutl, the FDR $<0.05$ was used as the threshold. The gene that was discovered by two software and more was identified as driver gene of ESCC. Used maftools (Mayakonda et al., 2018) to draw a mutation profile of the driver gene.

\section{Mutual Exclusion Analysis}

We used maftools to analyze gene exclusion and analysis, with a $p=0.05$ as a threshold.

\section{Clinical Correlation Analysis}

Generally, we divided cancer patients into two groups: young and old by the threshold value of age as 60. To improve the grouping method, we used the kmeans clustering method (https:/github.com/dstein64/kmeans1d) to divide the patients into two groups according to age. And the clustering was also based on 60 years old. The samples were divided respectively into two groups by the different clinical characteristics: T stage (T1-T2 VS T3-T4), TNM (S1-S2 VS S3S3), gender (male VS female), lymph node metastasis (YES VS NO), smoking (YES VS NO), drinking (YES VS NO), tumor grade (G1-G2 VS G3-G4). For the tumor location, the samples were divided into upper, middle, and lower three groups. And because there are only 6 samples of M1, so we do not analyze M stage. Clinical information of all ESCC were in Supplementary Table S3.

\section{Data Visualization}

We used ggscatterstats, ggwithinstats, venn, volcano graph, and histogram to draw related graphs on the hiplot platform (https:// hiplot.com.cn/), and used R ggplot2 package to plot other graphs.

\section{Statics Analysis}

The Fisher test was used to analyze the gene mutation with clinical characteristics, and the $t$-test was used to analyze the correlation between the gene mutation with numerical variables, such as age, aTMB, fTMB etc. 
For survival analysis, we used the survminer of the $\mathrm{R}$ package to analyze the survival differences between groups. For numerical variables, we use surv_cutpoint to determine the best cut point.

\section{Cell Culture Conditions}

The ESCC cell lines used in this study were from our lab. All ESCC cell lines were cultured in HyClone ${ }^{\mathrm{TM}}$ RPMI-1640 medium (GE Healthcare Life Sciences, HyClone Laboratories, Logan, UT, United States) with $10 \%$ fetal bovine serum (FBS; Gibco; Thermo Fisher Scientific, Inc., Waltham, MA, USA) at $37^{\circ} \mathrm{C}$ in a $5 \% \mathrm{CO}_{2}$ incubator. Replace the culture medium according to the cell status and subculture cells when the density of cells increases to about $80-90 \%$.

\section{Knockdown of KMT2D in ESCC Lines}

For the knockdown of KMT2D, we used the interfering RNAs (siRNAs) containing the sequence: $5^{\prime}$-GCACCATCATTCGGA ACGA-3' (KMT2D-sil) and 5' - CCAGTACTTTCGCTTCGAA $-3^{\prime}$ (KMT2D-si2). Cells at the logarithmic growth phase were transfected with siRNA using riboFECTTM CP Reagent (RIBOBIO, Guangzhou, China) according to the company's recommendation. The efficiency of knockdown was determined by real time-PCR.

\section{RNA Extraction and Real Time PCR}

Total RNA of ESCC cells was isolated by using RNAiso plus (Takara, Dalian, China). Complementary DNA (cDNA) was synthesized from $2 \mu$ g of total RNA using a PrimeScript ${ }^{\circledR}$ RT reagent Kit with gDNA Eraser (Takara). TB Green ${ }^{\circledR}$ Premix Ex $\mathrm{Taq}^{\circledR}$ II kit (Takara) was used in real time PCR. All real time PCR reactions were performed in triplicate with an Applied Biosystems Step One Plus (ABI, Foster City, CA, United States). The relative expression of target genes was determined by normalization to GAPDH expression and calculated using the $2^{-\Delta \Delta \mathrm{Ct}}$ formula. The primer sequences of GAPDH and KMT2D used in this study were listed in Supplementary Table S7.

\section{Cell Invasion and Migration Assay}

The invasion and migration ability of cells were detected using transwell plates $(8 \mu \mathrm{m}$, Corning, Inc.). For the migration assays, 50,000 to 80,000 cells/well were inoculated into the upper compartment of the transwell plate with serum-free culture. The lower part of the chamber was filled with culture fluid containing 10\% FBS. The upper surface cells are removed after $24 \mathrm{~h}$ or $48 \mathrm{~h}$. The cells passing through the membrane are fixed with $4 \%$ formaldehyde and stained with $0.1 \%$ crystal violet. Random five fields were chosen to count the number of transmigrated cells. For the invasion assays, the upper chambers were precoated with $100 \mu$ l Matrigel (1: 8 mixed with FBS-free media; BD Biosciences, Heidelberg, Germany) and proceeded as the same as described above.

\section{Cell Proliferation Assay}

In total, $5 \times 10^{3}$ transfected cells were seeded into each well of a 96-well plate in a final volume of $200 \mu \mathrm{l}$. After cultured for 24,48 , $72,96 \mathrm{~h}, 20 \mu \mathrm{l}$ CCK8 solution was added into each well, then the
TABLE 1 | ESCC cohort and samples.

\begin{tabular}{lcl}
\hline Year & ESCC sample number & \\
\hline 2014 & 88 & Journal \\
2014 & 113 & Nature \\
2015 & 104 & American Journal of Human Genetics \\
2016 & 144 & Gastroenterology \\
2017 & 96 & Nature \\
2017 & 92 & NATURE COMMUNICATIONS \\
2020 & 508 & Cell Research
\end{tabular}

cells were incubated at $37^{\circ} \mathrm{C}$ for $30 \mathrm{~min}$. The relative number of surviving cells in each group was measured by a spectrophotometer at $490 \mathrm{~nm}$.

\section{Colony Formation Assay}

Cells were seeded in a 6 -well plate at a density of 1,000 or 1,500 cells/well and incubated for 10 days at $37^{\circ} \mathrm{C}$ with $5 \% \mathrm{CO}_{2}$. After PBS cleaning, The cells were fixed with $4 \%$ polyformaldehyde for $30 \mathrm{~min}$ and stained with $1 \%$ crystal violet for $30 \mathrm{~min}$. The numbers of colonies containing more than 50 cells were counted under the microscope.

\section{RESULTS}

\section{ESCC Cohort and Samples}

Dozens of research cohorts have been published since 2014 (Supplementary Table S1) (Liu et al., 2017; Yu et al., 2019; Dutta et al., 2020; He et al., 2020; Mangalaparthi et al., 2020; Song et al., 2014; Cao et al., 2015; Zhang et al., 2015; Hu et al., 2016; Qin et al., 2016; Sawada et al., 2016; Chang et al., 2017; Chen et al., 2017; Dai et al., 2017; Du et al., 2017; Dai et al., 2018; Yan et al., 2019; Cui et al., 2020; Liu et al., 2020; Nature, 2017; Lin et al., 2014). In this study, we integrated and re-analyzed the 7 large cohorts including a total of 1,145 samples (Table 1) (Lin et al., 2014; Song et al., 2014; Zhang et al., 2015; Sawada et al., 2016; Chang et al., 2017; Nature, 2017; Cui et al., 2020). The whole analysis pipeline was shown in Figure 1A. There were 508 WGS samples and 637 WES samples a total of 149,085 mutations in the coding region (Supplementary Table S2). The median of ESCC tumor mutation burden (TMB) of nonsilent mutation in the coding region was located between Uterine Corpus Endometrial Carcinoma (UCES) and Liver hepatocellular carcinoma (LIHC), and was lower than Esophageal adenocarcinoma (EAC) (Supplementary Figure S1).

\section{Mutational Signatures in ESCC}

The previous analyses of ESCC mutation signatures of ESCC were all based on single base substitution (SBS). Here, we re-analyzed the SBS, double-base substitution (DBS), and small insertions and deletion (ID) signatures separately in both the coding regions of 1,145 ESCC samples and the whole genome of 508 ESCC using sigflow (Wang et al., 2020a). Total of 8 SBS, 9 DBS, 9 ID signatures were identified in the whole genome region (Figures 1B-D). 6 SBS and 2 DBS, and 3 ID signatures in the coding regions (Figures $2 \mathrm{~A}-\mathrm{C}$ ). Annotated with the COSMIC 


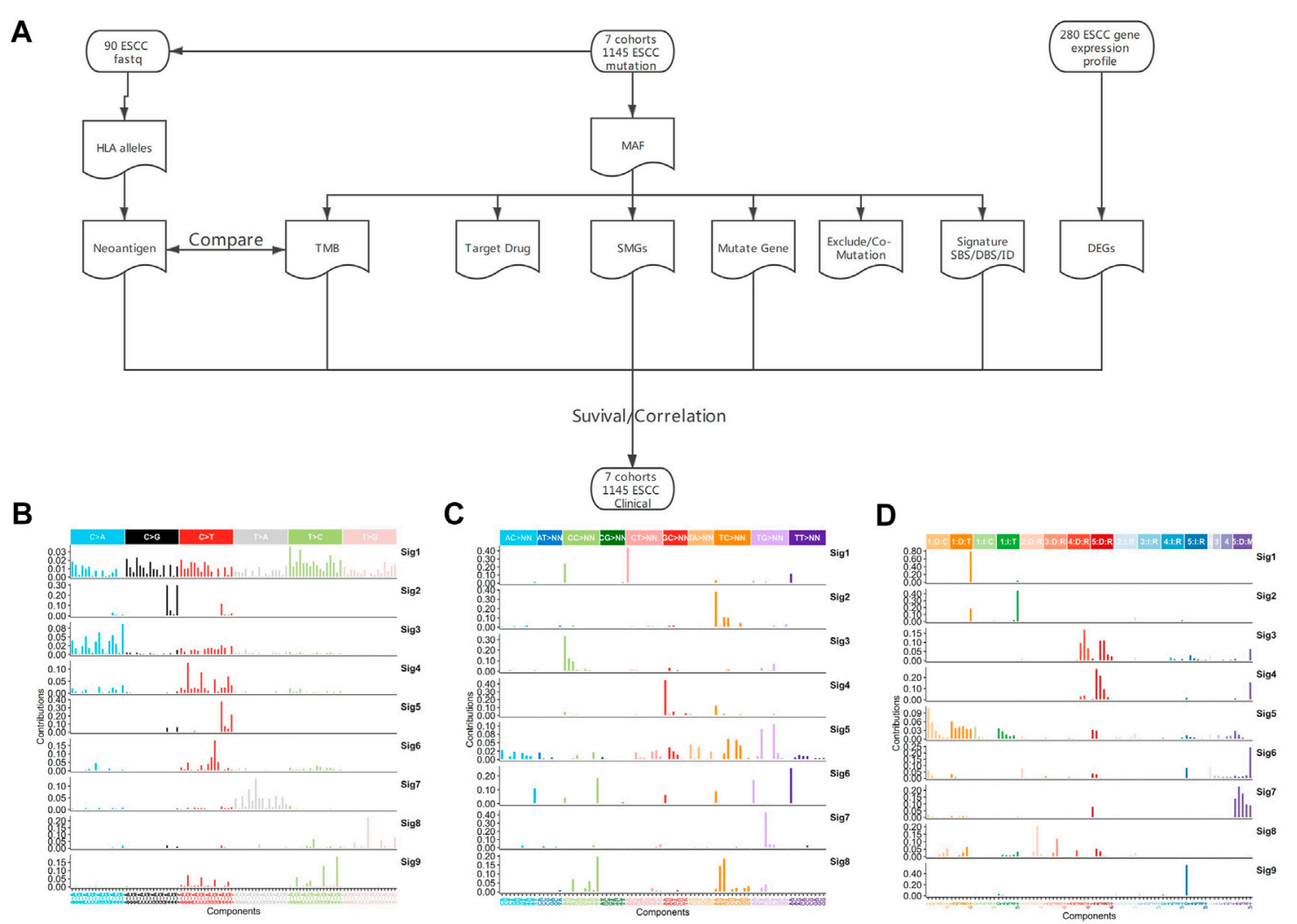

FIGURE 1 | Analysis pipeline and mutation signature of whole genome region in ESCC. (A) Analysis pipeline, (B) SBS signature of WGS, (C) DBS signature of WGS, (D) ID signature of WGS.

signatures, we found that the signatures in the coding region (Supplementary Table S4) and those in whole genome region (Supplementary Table S6) are similar in some aspects. A larger number of signatures were identified in our study. Besides that, two new signatures that have not been reported before were discovered in the whole genome region. One was WGS-SBS-S3 related to possibly damage by reactive oxygen species and the other was WGS-DBS-S1 related to prior chemotherapy treatment with platinum drugs. WGS-DBS-S1were correlated with the overall survival (OS) rates (Figure 2D, $p=0.00044$ ). Next, we analyzed the correlation between signatures of coding regions and clinical characteristics (Figure 2E, Supplementary Table S5) or the OS rates. We found that most of them were related to drinking, smoking, gender and lymph metastasis (FDR $<0.05)$. WES-SBS-S6 (Figure 2F, $p=0.00044$ ) and WES-ID-Sig1 (Figure 2G, $p=0.00044$ ) were correlated with the OS rates.

\section{Tumor Mutation Burden in ESCC}

$\mathrm{TMB}$ is an important cancer immunotherapy biomarker (Boumber, 2018a; Chan et al., 2019; Jardim et al., 2020). Adult and pediatric patients with high TMB ( $\geq 10$ mutations/megabase) were approval for pembrolizumab by FDA (Subbiah et al., 2020). Currently, there is not a standard way to calculate TMB. Some studies excluded synonymous mutations (Zehir et al., 2017), while some studies include synonymous mutations ( $\mathrm{Xu}$ et al.,
2019). In this study, we defined aTMB which includes synonymous mutations and fTMB which excludes synonymous mutations. The average value of aTMB was 4.30 in the 1145 ESCC patients, and the median value of aTMB was 3.58 in the 1145 ESCC patients. The average value of fTMB was 3.27 in the 1145 ESCC patients, and the median value of aTMB was 2.75 in the 1145 ESCC patients. The consistency between aTMB and $\mathrm{fTMB}$ in ESCC was significant (Figure 3A, R $=1, p=$ 0 ), but the values calculated by the two methods are significantly different (Figure 3B, $p=4.13 \mathrm{e}-13$ ). Thus, we speculated that the calculation method needed to be considered when using a specific threshold.

We compared different group OS rates using different TMB values. If grouping patients with the TMB value 10 (Cui et al., 2020), there was no significant difference between the high TMB group and the low TMB group regardless of using aTMB (Figure 3C, $p=0.14$ ) or fTMB (Figure 3D, $p=0.43$ ) method. However, using survminer (Kassambara et al., 2017), the optimal grouping point was automatically selected by the surv_cutpoint method. Using the aTMB value 8.0 as the threshold, we found that there was a significant difference OS rates between the high and low TMB groups (Figure 3E, $p=0.014$ ). There was also significant difference between the high and low fTMB groups (fTMB >5.7, Figure 3F, $p=0.04$ ). Therefore, different analysis methods require the corresponding TMB threshold values. In 


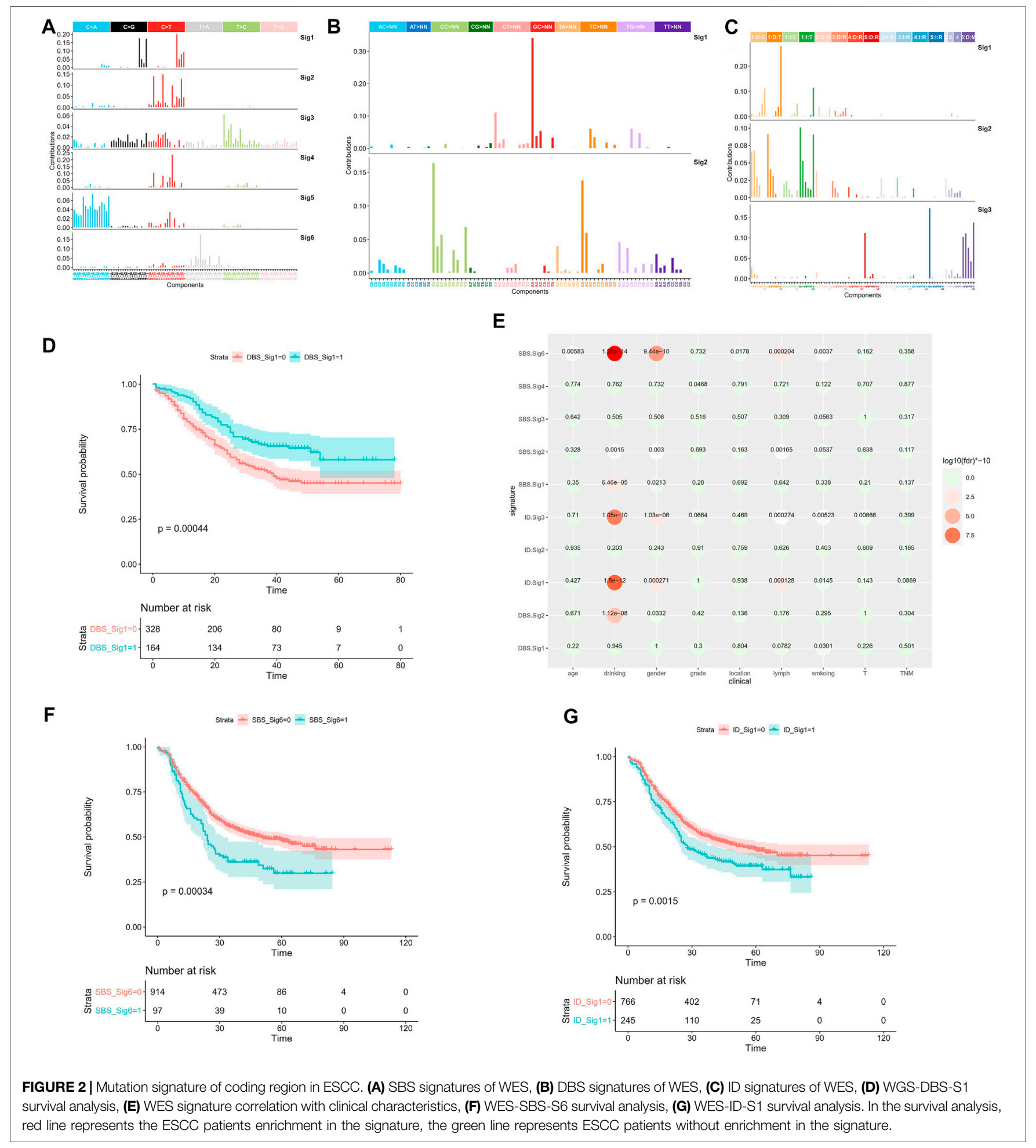

addition, the commonly defined high-TMB threshold value should not be applied in different cancer types. For ESCC, we thought the aTMB as 8.0 was better than 10 . And fTMB as 5.7 was recommended for the similar study. However, the accurate value of the high TMB threshold suitable for clinical grouping requires further validation.
High Heterogeneity of Neoantigen in ESCC Neoantigens play an important role in tumor immunotherapy. We analyzed the neoantigens of 90 WES samples (Zhang et al., 2015) with pvactools (Hundal et al., 2020) for obtaining fastq data. 2 new neoantigen indicators were defined as follows. One was the tumor neoantigen burden (TNB), which was the number 


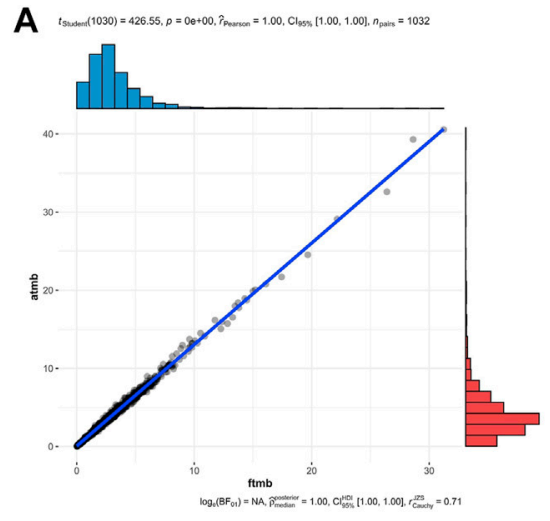

C
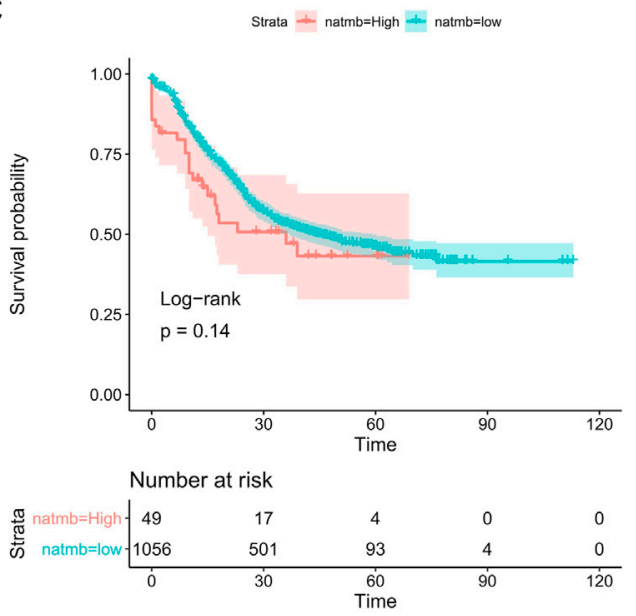

E

Strata + natmb2=High + natmb2=low
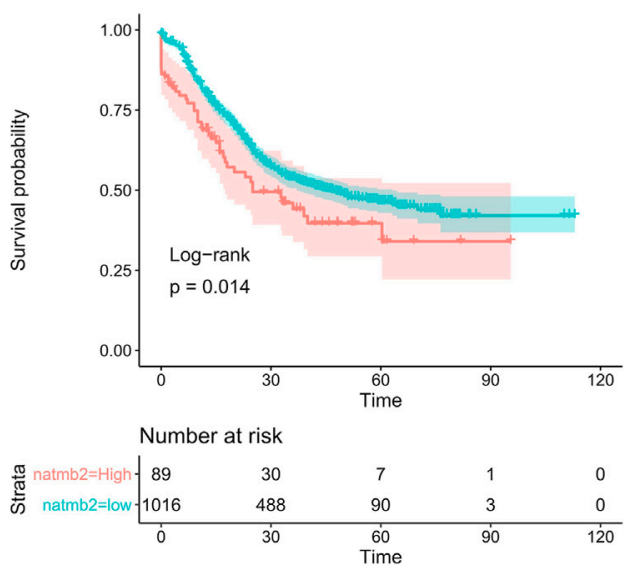

B

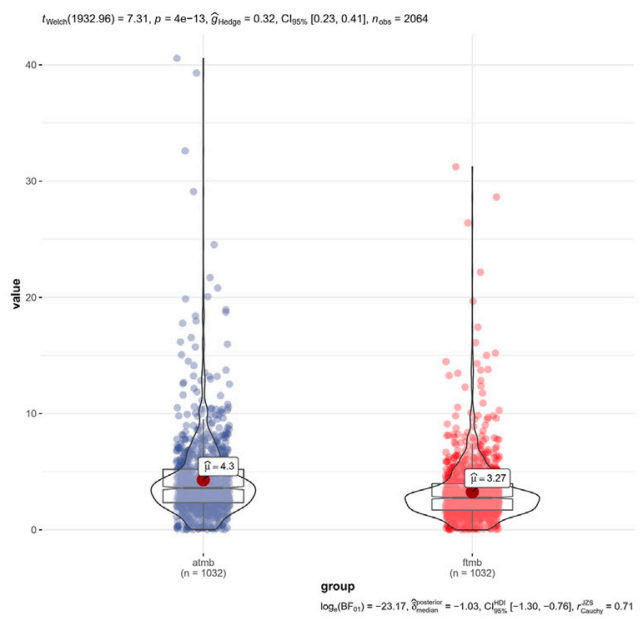

D Strata $\perp$ nftmb=High $\perp$ nftmb=low
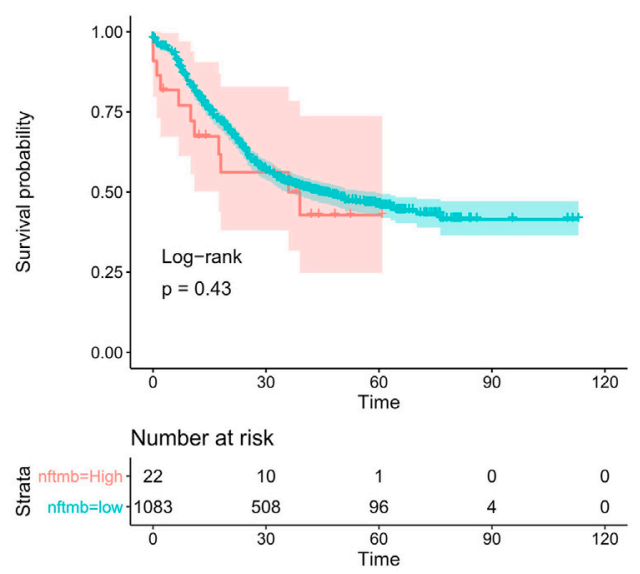

F $\quad$ Strata \pm ttmb=high \pm ftmb=low
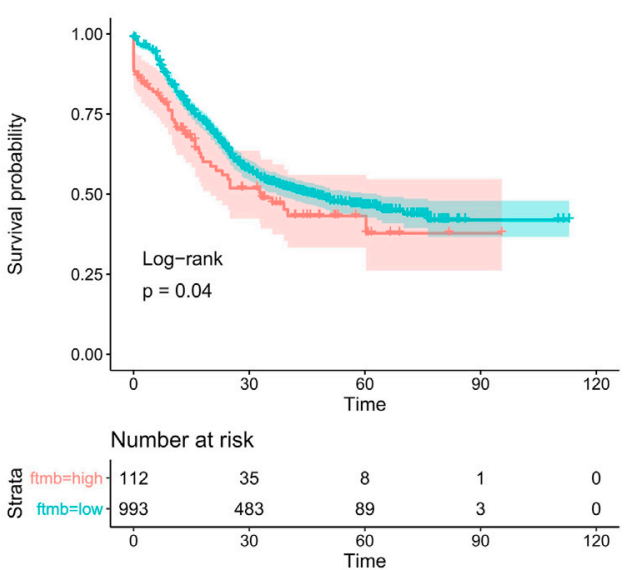

FIGURE 3 ITMB in ESCC. (A) the correlation between aTMB and TTMB, (B) compared the mean value between aTMB and fTMB, (C) Survival analysis of aTMB (high aTMB >10), (D) Survival analysis of fTMB (high fTMB >10), (E) Survival analysis of aTMB (high aTMB >8.0), (F) Survival analysis of fTMB (high fTMB >5.7).

of neoantigen in the sample. The other was the tumor neoantigen score (TNS), which was the score of neoantigen in the sample (See methods).
The correlation between TNS and TMB was very high (Figure 4A, $\mathrm{R}=0.85, p=6.35 \mathrm{e}-26$ ), and the correlation between TNB and fTMB was also high (Figure $4 \mathbf{B}, \mathrm{R}=0.81$, 

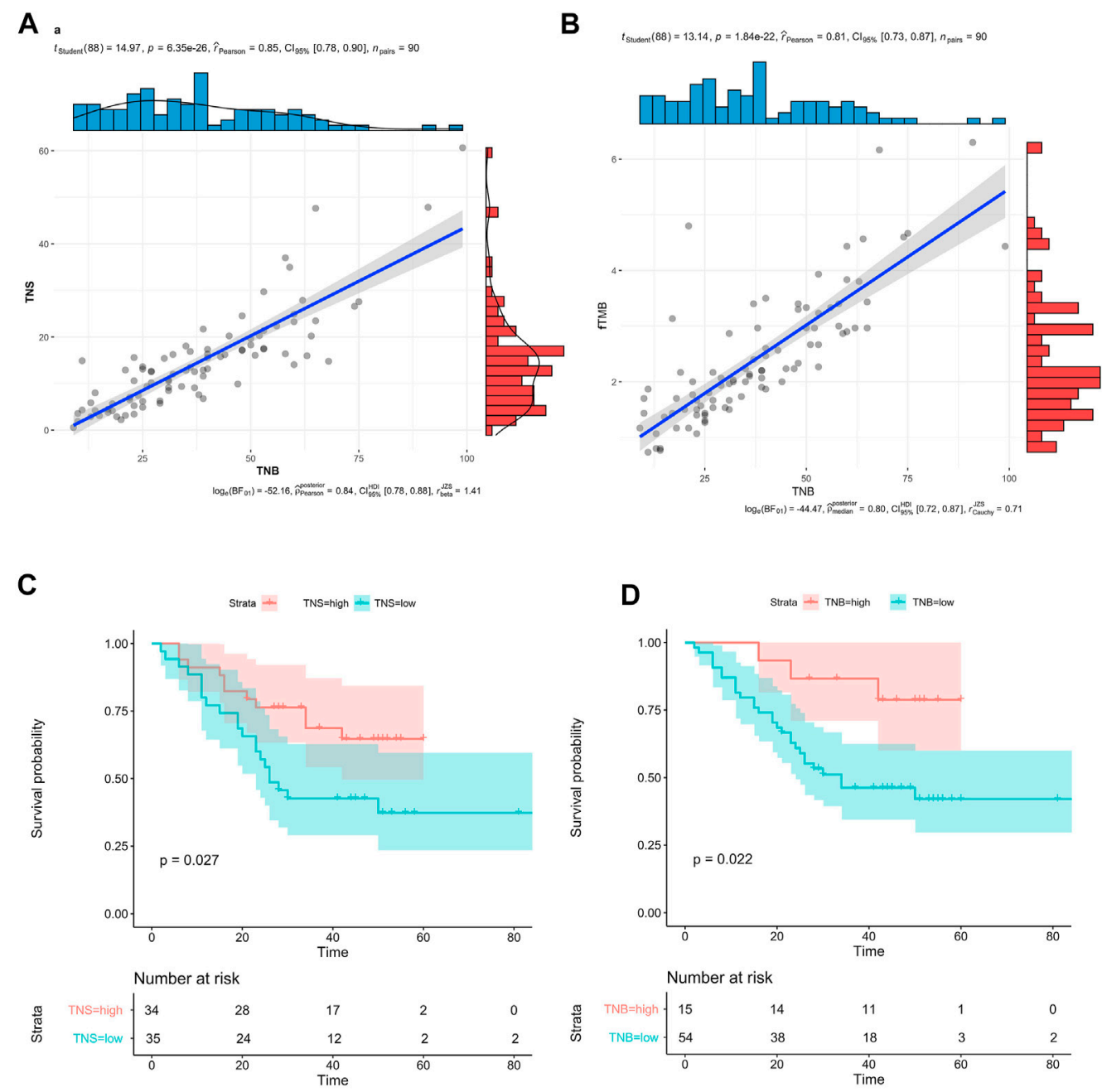

E
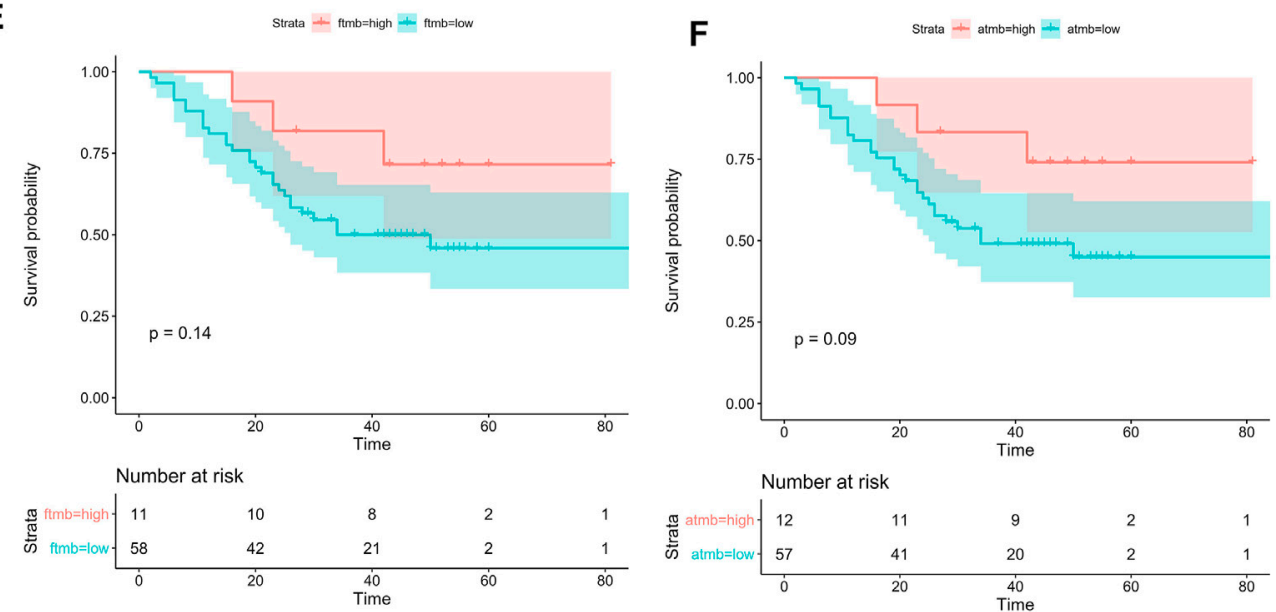

FIGURE 4 | Neoantigen in ESCC. (A) The correlation bewtten TNB and TNS, (B) Compared the mean value between TNB and fTMB, (C) Survival analysis of TNS (high TNS >2.2), (D) Survival analysis of TNB (high TNB>2.5), (E) Survival analysis of fTMB (high fTMB >1.6), (F) Survival analysis aTMB (high aTMB >1.8).

$p=1.84 \mathrm{e}-22)$. Since there were no mutation hotspots in ESCC, the highest mutation frequency was TP53:p.R282W (2.41\%), so the neoantigens produced by patients are different. The highest frequency of the neoantigen peptides can only be found in 3 patients. Only 2 of this neoantigen accounted for $0.6 \%$ of all neoantigens. 60 neoantigens appeared in 2 patients accounted for 
A

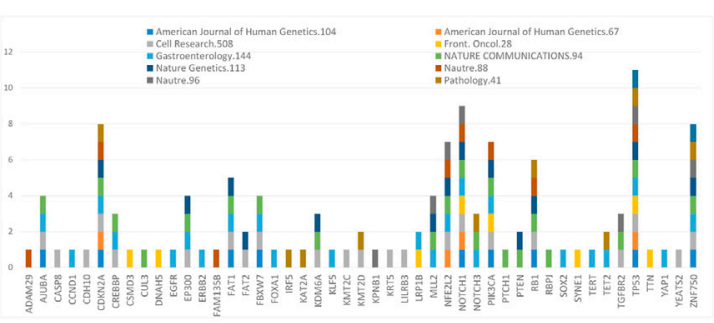

C

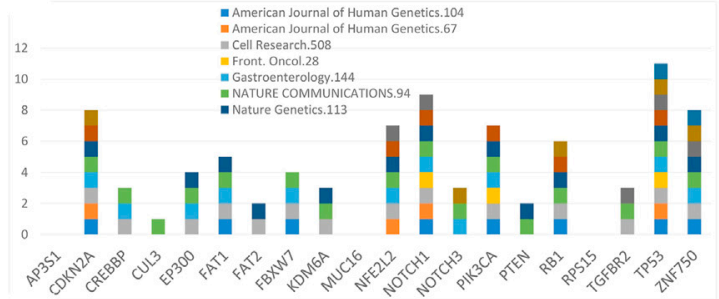

D

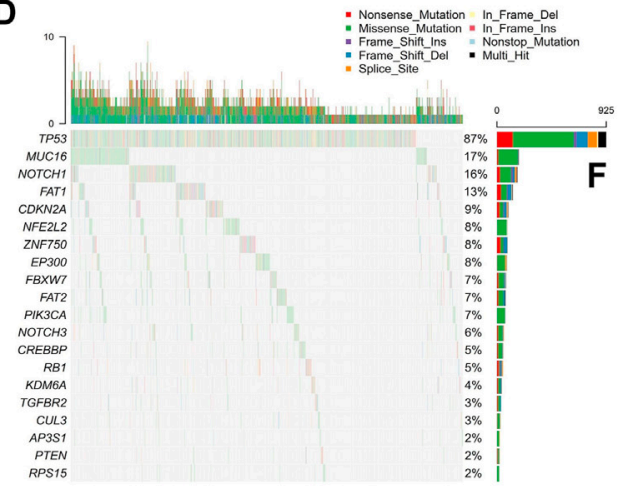

$\mathrm{H}$

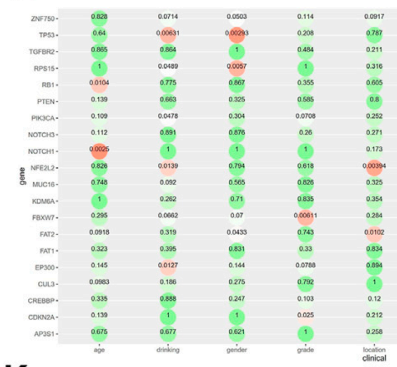

K

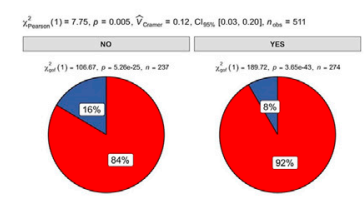

(1)

L
B

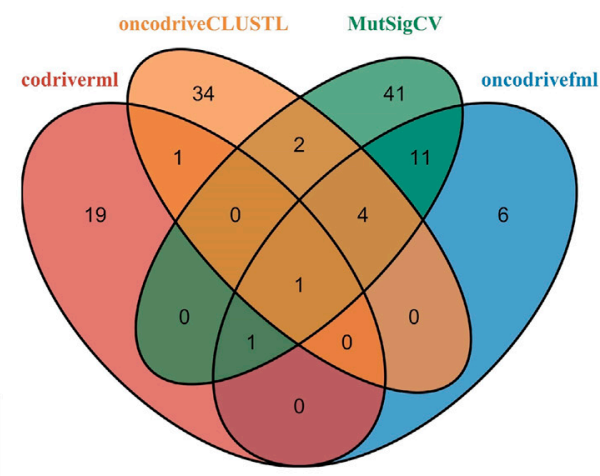

E

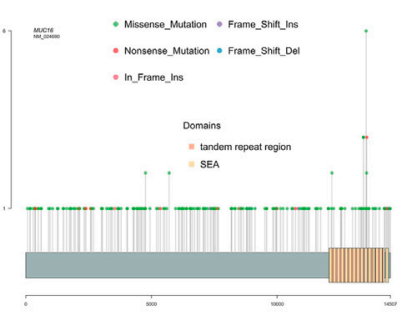

G

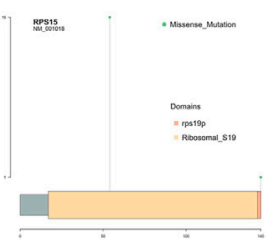

I

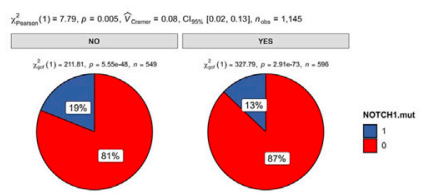

J
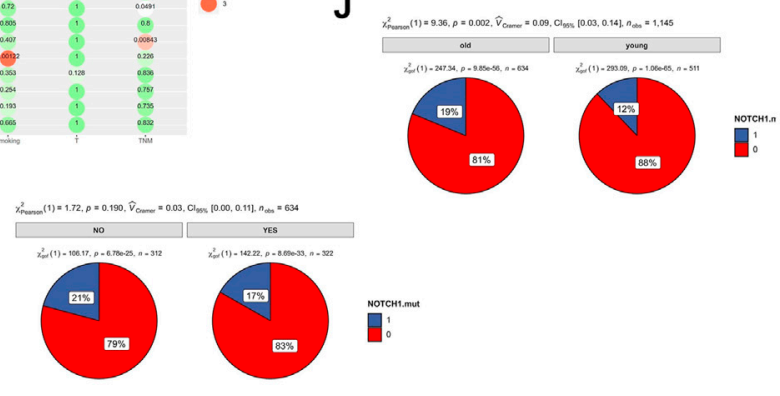

FIGURE 5 |SMGs in ESCC. (A) SMGs of ESCC in previous studies, (B) SMGs of ESCC identified by 4 software, (C) The distribution of SMGs of ESCC identified by 4 software in previous studies, (D) Mutation profile of SMGs in ESCC, (E) MUC16 mutation in ESCC, (F) AP3S1 mutation in ESCC, (G) RPS15 mutation in ESCC, (H) The correlation between SMGs of ESCC and clinical characteristics, (I) The correlation between NOTCH1 mutation and lymph node metastasis of ESCC, (J) The correlation between NOTCH1 mutation and different age group of ESCC patients (young and old), (K) The correlation between NOTCH1 mutation and lymph node metastasis of young ESCC patients (age<60), (L) The correlation between NOTCH1 and lymph node metastasis of old ESCC patients (age >=60). 
$1.8 \%$. The proportion of neoantigens that appeared in one patient was $97.6 \%$. Therefore, we thought that the effective cancer vaccine is hard to be developed for ESCC based on our results.

The 90 patients were divided into 2 groups based on the TNB or the TNS, by the surv_cutpoint method in survminer. There were significant differences between the high and low groups (TNS $>2.2, p=0.027$, Figure 4C; TNB $>2.5, p=0.022$, Figure 4D). Compared with the TNB high group, the high group of TNS had more patients, the ratio of the high-low group is to $34: 35$, and the ratio is $15: 54$ in the TNB.

Compared with neoantigen, 90 patients were divided into 2 groups based on the fTMB (high fTMB $>1.6$ ) or aTMB (high aTMB $>1.8$ ), by the surv_cutpoint method in survminer. There was no significant difference between the high and low groups (Figure 4E, $p=0.14$, Figure 4F, $p=0.09$ ). Compared with the OS, neoantigen was a better biomarker than TMB. And the TNS was a suitable factor for neoantigen calculation compared with the TNB.

\section{Potential Targeted Therapy Drugs in ESCC}

At present, limited targeted drugs have been approved for ESCC. In order to find potential targeted drugs for ESCC, we searched the targeted drug databases OncoKB (Chakravarty et al., 2017) and Civic (Griffith et al., 2017). Currently, there were no special targeted drugs for ESCC in the OncoKB database. In the Civic database, the targeted drugs for ECA and ESCC were different, but the highest evidence level was only B for ESCC. Recently, some studies have begun to treat different cancer patients with specific targeted drugs based on the same gene mutation position (Gambacorti-Passerini et al., 2018). Our study uses the OncoKB and Civic databases for analysis of targeted drugs for particular gene mutation in ESCC. We found that there are only drugs targeting only TP53 and PIK3CA gene mutations (Supplementary Figure S4). Among them, 135 patients, accounting for $11.8 \%$, can benefit from the drug AMGMDS3 targeting TP53 gene (Supplementary Figure S4). From the data of the two databases, patients with ESCC can benefit little from the existing targeted drugs. More research is required to find new targeted drugs in the future.

\section{Significant Mutated Genes in ESCC}

We re-analyzed more than 20 sequencing datasets (Liu et al., 2017; Yu et al., 2019; Dutta et al., 2020; He et al., 2020; Mangalaparthi et al., 2020; Song et al., 2014; Cao et al., 2015; Zhang et al., 2015; Hu et al., 2016; Qin et al., 2016; Sawada et al., 2016; Chang et al., 2017; Chen et al., 2017; Dai et al., 2017; Du et al., 2017; Dai et al., 2018; Yan et al., 2019; Cui et al., 2020; Liu et al., 2020; Nature, 2017; Lin et al., 2014) of ESCC and found that only 11 studies included the driver gene analyses (Supplementary Table S1). Seven of them used MutSigCV for analysis (Lawrence et al., 2013), dNdScv was used in one study (Martincorena et al., 2017), and MutSigCV and oncodriveFML were applied together in another study (Mularoni et al., 2016). A total of 47 driver genes were found in 11 published studies. Among them, 7 genes (TP53 (11), NOTCH1 (9), CDKN2A (8), ZNF750 (8), NFE2L2 (7), PIK3CA (7), RB1 (6)) were considered driver genes in more than 5 studies and 26 genes were considered driver genes in only one study (Figure 5A). Previous studies were limited because of using fewer analysis tools or the relatively small sample size. Therefore, the reported driver genes identified by previous studies is limited.

To identify the driver genes of ESCC in a more accurate and comprehensive way, four software were applied in our study: MutSigCV, driverml (Han et al., 2019), OncodriveFML, and OncodriveCLUSTL (Arnedo-Pac et al., 2019). A total of 20 driver candidate genes were identified (Figure 5B, Figure 5C). Among them, TP53 was a driver gene recognized by all software. EP300, FAT1, FBXW7, PIK3CA, ZNF750, and AP3S1 were identified as driver genes in three software. Among the 20 driver genes, 14 genes were reported in three or more studies, FAT2 and PTEN were reported as driver genes in only 2 studies, and CUL3 was reported as driver gene in only 1 study. AP3S1, MUC16, RPS15 had not been reported as a driver gene (Figure 5C). AP3S1, MUC16, and RPS15 were three newly discovered driver genes. The MUC16 had been considered as a driver gene in the pan-cancer driver gene research (MartínezJiménez et al., 2020), the mutation frequency of MUC16 in ESCC was high (17\%, Figure 5D), and there was not hotspot mutation in the MUC16 (Figure 5E). while AP3S1 and RPS15 had not been reported as driver genes before, and there were hotspot mutations in the AP3S1 (Figure 5F) and RPS15 (Figure 5G) in the ESCC, the mutation frequency of AP3S1 and RPS15 were low in the TCGA, and there was not hotspot mutation in the TCGA (Supplementary Figures S2, S3).

Next, we analyzed the correlation between driver gene and clinical characteristics (Figure 5H). The results showed that some genes were related to multiple clinical characteristics, such as ZNF750 related to lymph node metastasis and TNM stage. NOTCH1 mutated were related lymph node metastasis $(p=$ 0.005 , Figure 5I) and related to age ( $p=0.002$, Figure 5J). Moreover, we found that NOTCH1 mutated was related to young ( $p=0.005$, Figure 5K), not to old ( $p=0.190$, Figure 5L).

We analyzed the mutual exclusion and co-mutation of all mutant genes (Supplementary Figure S5), and found that ZNF750 and CDKN2A was mutually exclusive $(p=$ $0.004809937)$. A total of 52 pairs of genes have been found to have co-mutations, the most significant of which are TTN and IFSIP2 $(p=2.94 \mathrm{E}-05)$.

\section{Correlation Between Gene Mutation and Lymph Node Metastasis}

Most cancer death was originally caused by metastasis. Tumor metastasis was mainly included lymph node metastasis and blood metastasis. In our study, there were 52\% ESCC patients with lymph node metastasis (Figure 6A). And the survival time of ESCC patients with lymph node metastasis was lower than that of ESCC patients without lymph node metastasis $(p<0.0001$, Figure 6B). We found that the drinking history, smoking history, $\mathrm{T}$ stage, tumor location and tumor grade were related to lymph node metastasis $(p<0.05)$. It was worth noting that among these clinical characteristics, tobacco consumption largely promoted the increase in the frequency of lymph node metastasis. Compared with non-smoking patients, the frequency of lymph 
A $\chi_{\text {gof }}^{2}(1)=1.93, p=0.165, \widehat{v}_{\text {cramer }}=0.03, \mathrm{Cl}_{95 \%}[0.00,0.09], n_{\text {obs }}=1145$

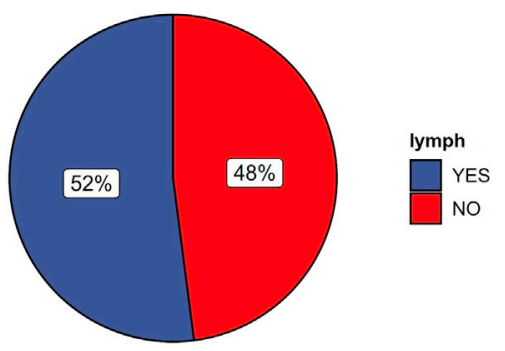

$\log _{\mathrm{e}}\left(\mathrm{BF}_{01}\right)=2.75, a_{\text {Gunel-Dickey }}=1.00$

C
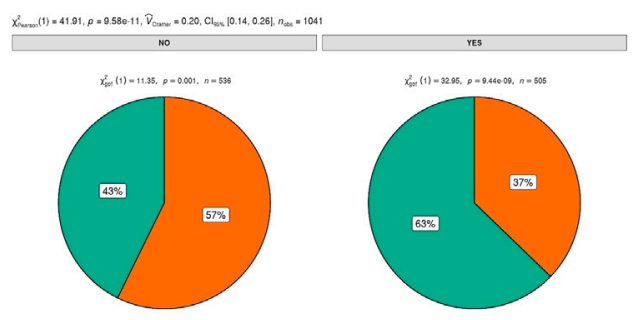

$\underset{\substack{\text { NO } \\ \text { Nes }}}{\text { vymph }}$

E

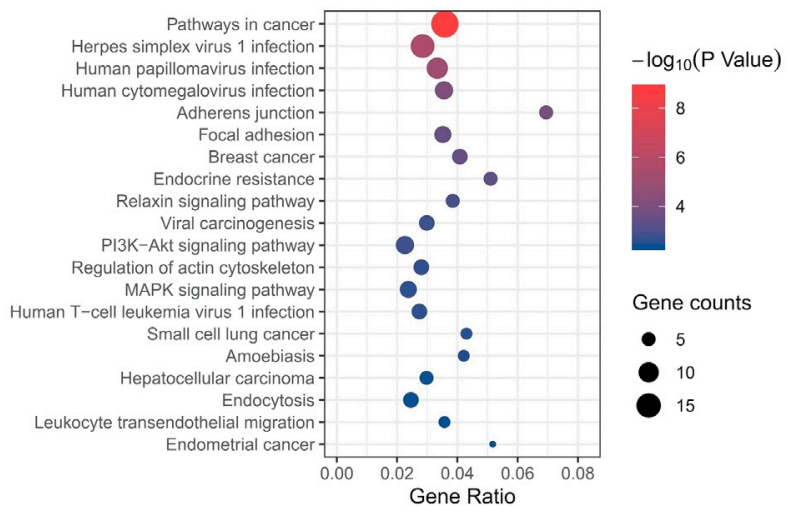

B
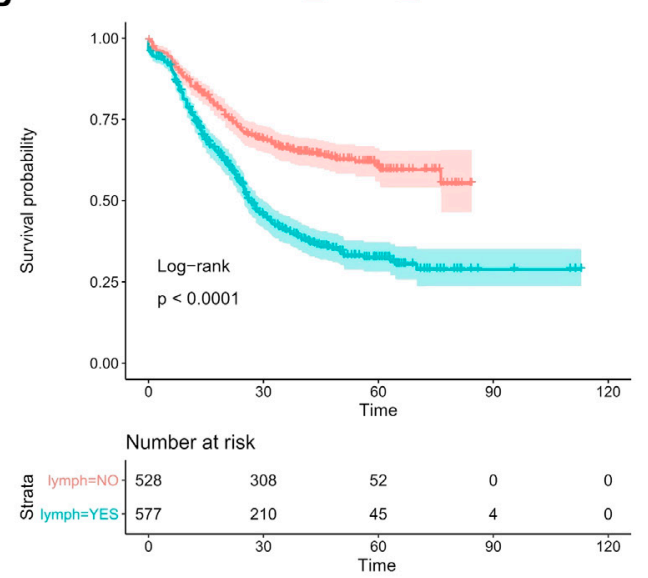

D
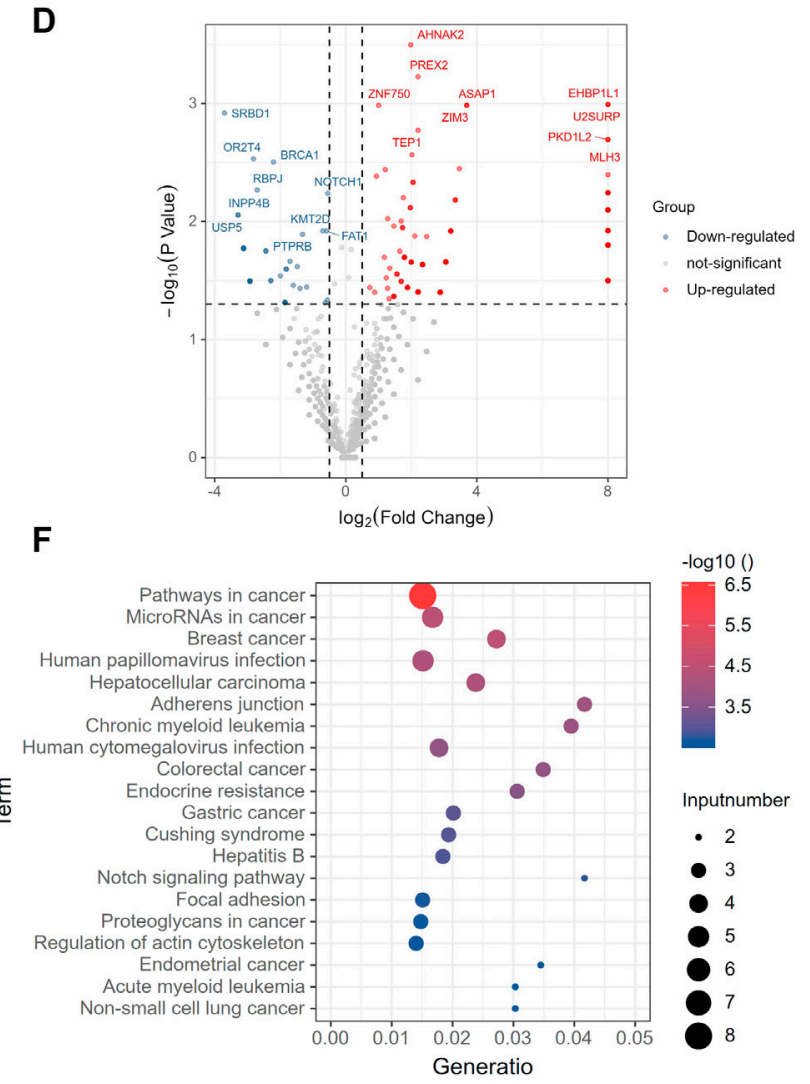

FIGURE 6 | Gene mutation with lymph node metastasis. (A) the frequency of lymph node metastasis in ESCC, (B) Survival analysis of lymph node metastasis in ESCC, (C) smoking related with lymph node metastasis in ESCC, (D) volcano plot of the correlation gene mutation with lymph node metastasis, $x$-axis was the log ${ }_{2}$ (the ratio of mutated rate of lymph node metastasis group and the mutated rate of without lymph node metastasis group), and $y$-axis was $P$ (the correlation gene mutation with lymph node metastasis), (E) KEGG enrichment pathway of the higher mutation gene of lymph node metastasis compared with no lymph node metastasis, (F) KEGG enrichment pathway of the lower mutation gene of lymph node metastasis compared with no lymph node metastasis.

node metastasis was $43 \%$, rising to $63 \%(p=9.58 \mathrm{E}-11$, Figure 6C).

There were 237 mutated genes related with lymph node metastasis $(p<0.05)$. Among them, 188 genes showed higher mutation rate of lymph node metastasis than no lymph node metastasis group, called "higher mutated genes (HMG)". These gene mutations may promote lymph node metastasis. While 49 genes showed lower mutation rate in lymph node metastasis group than that in no lymph node metastasis group, called "lower mutated gene (LMG)" (Figure 6D). And these gene mutations may prevent lymph node metastasis. We used kobas (Xie et al., 2011) respectively to do KEGG pathway enrichment analysis of 


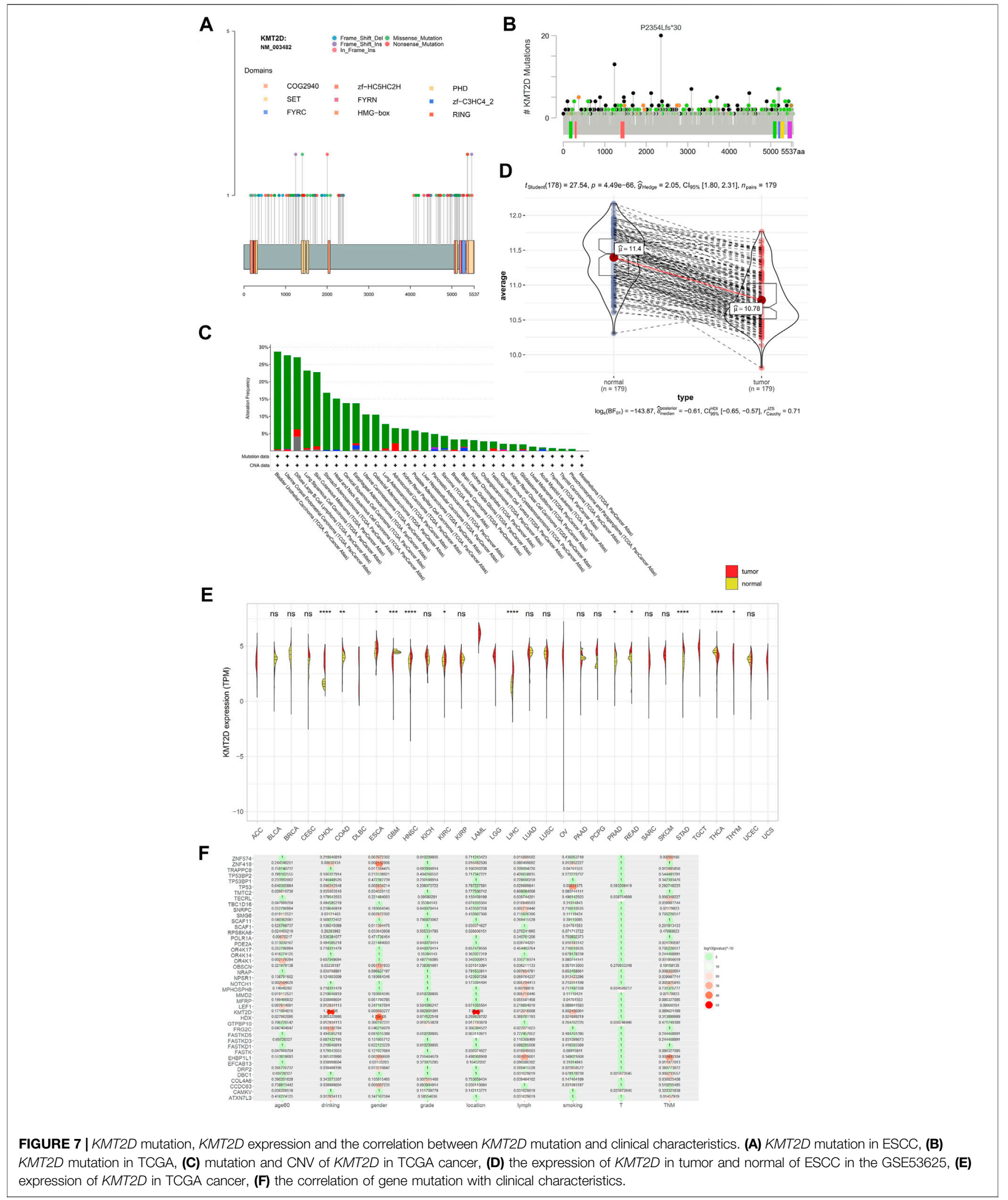


A

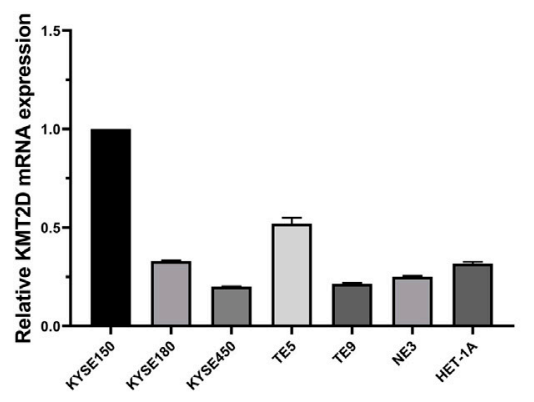

C

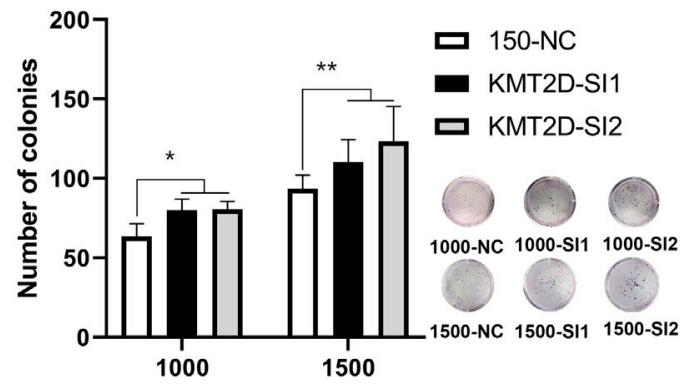

E

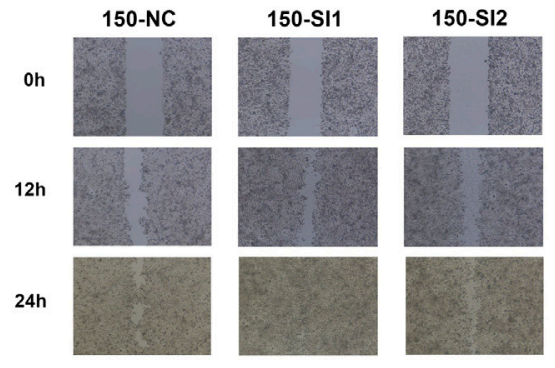

F

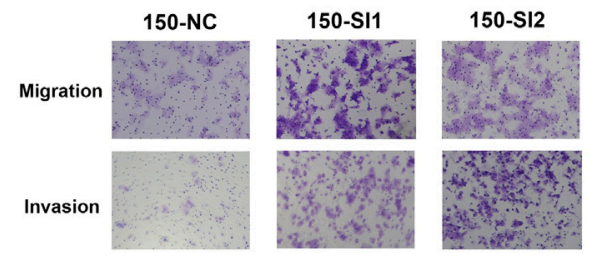

B

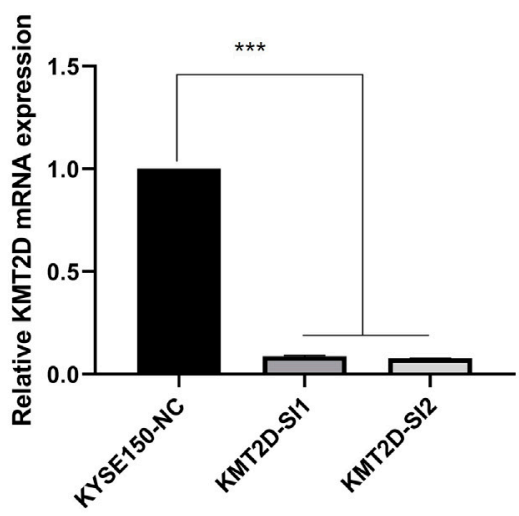

D
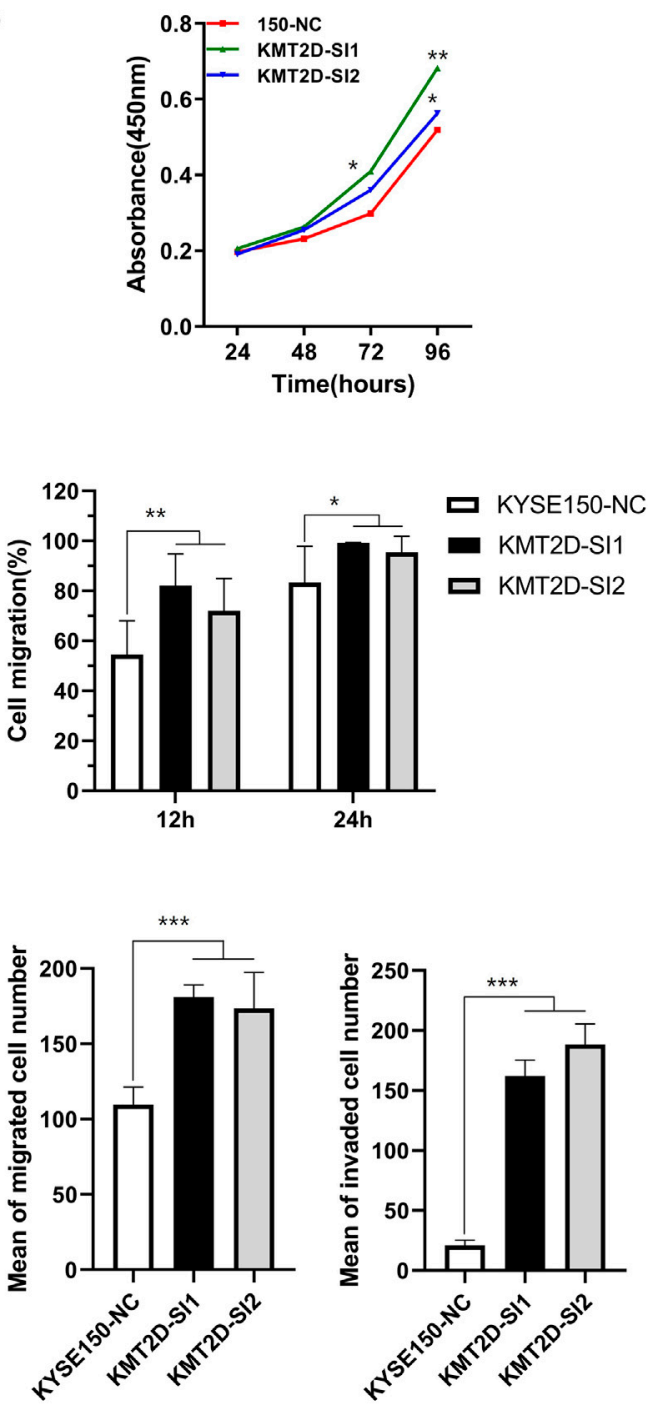

FIGURE 8| The effect of KMT2D in KYSE150 cell line. (A) The mRNA expression of KMT2D in normal esophageal epithelial cell lines and ESCC cell lines detected by q-RTPCR, (B) The knockdown efficiency of KMT2D in KYSE150 cell line was verified by q-RTPCR, (C) KMT2D knockdown promoted the ability of colony formation in KYSE150 cell line compared to the control, (D) KMT2D knockdown promoted the ability of proliferation in KYSE150 cell line compared to the control, (E) KMT2D knockdown promoted the ability of migration in KYSE150 cell line compared to the control, (F) KMT2D knockdown promoted the ability of migration and invasion in KYSE150 cell line compared to the control. All data are presented as the mean \pm standard deviation of the three in-dependent experiments. 
these two types of genes. We found that the Pathways in cancer, Herpes simplex virus 1 infection, Human papillomavirus infection were the top 3 pathways in enrichment of those LHG (Figure 6E). And pathways in cancer, MicroRNAs in cancer, Breast cancer were the top 3 pathways of those LWG (Figure 6F).

\section{KMT2D Plays a Tumor Suppressor Role in ESCC}

The frequency of KMT2D mutation was $15.02 \%$ (top 3 of all mutated gene), and there was no hotspot mutation in this gene mutation in ESCC (Figure 7A) and other cancer (Figure 7B). The KMT2D mutation frequency was highest in BLCA closed to $30 \%$ (Figure 7C). The expression of KMT2D in tumor was lower than that in normal tissues in the GSE53625 (Figure 7D). The different expression pattern between normal and tumor tissues existed in many cancer types in the TCGA data (Figure 7E). We analyzed the correlation between gene mutation and clinical characteristics (Figure 7F). KMT2D mutation was related to multiple clinical characteristics (drinking, location, lymph, gender, and smoking, top1 of the number with gene-related clinical characteristics). To verify the biological role of KMT2D in ESCC, we compared the expression levels of KMT2D in several cell lines including normal esophageal epithelial cells such as NE3, HET-1A and ESCC cell lines such as KYSE150, KYSE180, KYSE450, TE-5, and TE-9. The expression levels of KMD2T in different cell lines were shown in Figure 8A. We selected KYSE150 cell line with a relatively high endogenous level expression for perform knockdown experiments. Knockdown of KMD2T in KYSE150 cell line showed a significant decrease (Figure 8B). The results of CCK8 and the colony formation assays showed that the cell proliferation, colony formation ability was significantly improved in KMT2D knockdown group compared with the control group (Figures 8C,D). Besides that, the knockdown of KMT2D can markedly promote cell invasion and migration of KYSE150 (Figures 8E,F).

\section{DISCUSSION}

Mutation signature has always been an important part of cancer research. The analysis of mutation signature has also evolved from SBS to DBS, ID and other complex mutations (Alexandrov et al., 2020). In this study, for the first time, the SBS and ID of ESCC were analyzed. We identified 8 SBS, 9 DBS and 9 ID signatures in the whole genome region. And We also found 6 SBS,2 DBS, and 3 ID signatures the coding region. Most signatures in WGS and WES were similar in ESCC, for example WES-SBS-S1, WGS-SBS-S1 and WGS-SBS-S5 were correlation with APOBEC. However, in the whole genome region, there were more signatures and new signatures identified. For example, WGS-DBS-S1 correlation with prior chemotherapy treatment with platinum drugs, which cannot be identified in the coding region. The mutation signatures in whole genome region can provide more valuable information compared with coding region. This not only deepens our understanding of the mutational signature of ESCC, but also enriches the number of mutational signatures of ESCC. And we also found two new mutation signatures. These mutation signatures can not only be used for the identification of ESCC, but also can be used as new factors for related machine learning or deep learning.

Using a larger size of samples and various driver gene identify software will be helpful in determining the accurate driver genes of ESCC. In our study, we identified 20 drivers using the mutation data of 1,145 samples by using 4 software and 3 (AP3S1, MUC16, RPS15) of them were discovered for the first time. The mutation frequency of MUC16 in ESCC was high. MUC16was recognized as a driver gene in the pan-cancer driver gene research (MartínezJiménez et al., 2020). MUC16 was reported as an important biomarker for cancer (Felder et al., 2014a; Aithal et al., 2018). According to studies in other cancers (Felder et al., 2014b; Aithal et al., 2018; Yeku et al., 2020; Yu et al., 2020), MUC16 may also be an important immunotherapy target for ESCC. The mutation frequency of AP3S1and RPS15 were low both in ESCC and TCGA, but hotspot mutation was found in the ESCC. Even with hotspot mutations in the two gene but too low mutation frequency limits their value in clinical applications.

We tried to find better immunotherapy and targeted therapy targets for ESCC according to the results obtained from mutations of multiple cohorts. From the OncoKB and Civic databases, there was no targeted therapy drug for ESCC. Both TMB and neoantigen were new immunotherapy markers (Schumacher and Schreiber, 2015; Lu and Robbins, 2016; Łuksza et al., 2017; Boumber, 2018b; Samstein et al., 2019; Zhou et al., 2020; Yin et al., 2021). In the 7 cohorts, the TMB was analysis in only one cohort (508 ESCC patients, published in 2020 years on Cell Research). In this cohort more than 10 mutations per Mb was as TMB-H. TMB was attracted more and more attention by researchers. In recent years, researchers have carried out some systematic research on related immunotherapy on TMB. As a continuous variable, TMB has no recognized cutoff suitable for all cancers. TMB cutoff values is supposed to be different in various cancer types. For ESCC, there is no relevant research to determine the appropriate TMB cutoff. For ESCC, the conventional value for TMB was slightly higher. It may be more appropriate to use the aTMB as 8 , and it was more appropriate to use the fTMB as 5.7. The threshold of $\mathrm{TTMB}$ and aTMB were caculated by the surv_cutpoint method of "survminer" R package (Kassambara et al., 2017). This method was widely used in various cancers survival analysis for continuous variable (Zhou et al., 2019; Wang et al., 2020b; Lai et al., 2020; Wu et al., 2020). But we need more research to determine the true suitable threshold of TMB. Only a few somatic mutations will produce peptides that have been properly processed and loaded onto the MHC complex. Generally, the more somatic mutations a tumor has, the more likely it is to form a neoantigen (Coulie et al., 2014; Snyder and Chan, 2015). In the 90 WES ESCC cohort, we found that compared with TMB, neoantigen was related to the OS. In ESCC, compared with $\mathrm{TMB}$, neoantigen may be a better marker, however, due to the limitation of sample size, further research is needed. In the relationship between $\mathrm{TMB}$ and prognosis, we observed the opposite phenomenon. High TMB had a poor prognosis in 1,145 samples, but in 90 WES samples, high TMB had a good prognosis. This may be due to the number of samples. Similar phenomena have also been found in lung cancer research 
(Greillier et al., 2018; Alborelli et al., 2020; Nie et al., 2020). Neoantigen therapy is a highly individualized tumor immunotherapy, different patients often have different neoantigens for immunotherapy (Türeci et al., 2018; Blass and Ott, 2021; Jou et al., 2021). For ESCC, neoantigen presents high heterogeneity. Thus, personalized immunotherapy targeting to neoantigens may be a promising treatment for ESCC patients.

Lymph node metastasis is an essential cause of death in cancer (Chaffer and Weinberg, 2011). We analyzed the gene mutation with different clinical characteristics and focused on analyzing the gene mutations with or without lymph node metastasis. Therefore, the identification of biomarkers in ESCC lymph node metastasis will greatly help ESCC treatment and prognosis. Some gene mutations promoted lymph node metastasis, while other gene mutations inhibited lymph node metastasis. We have identified these two types of genes and performed an enrichment pathway analysis, which could help us to understand the mechanism of lymph node metastasis in ESCC. We found that NOTCH1 mutations were only associated with lymph node metastasis in young ESCC patients, which may be used for the diagnosis of early lymph node metastasis in ESCC.

We found that KMT2D (Lysine methyltransferase 2D) was related to multiple clinical characteristics and this gene had a relatively high mutation frequency. KMT2D was a gene of histone methyltransferase that methylates the Lys-4 position of histone H3, and there some research in other cancers (Guo et al., 2013; Toska et al., 2017; Xiong et al., 2018; Li et al., 2019; Sun et al., 2019; Alam et al., 2020; Zheng et al., 2021), but there was still a lack of reports in ESCC (Abudureheman et al., 2018). Knockdown of this gene in ESCC cell lines increased invasion and migration ability. The results indicated that KMT2D may plays an important role in ESCC. This gene can be used as a potential target for ESCC treatment.

\section{REFERENCES}

Abudureheman, A., Ainiwaer, J., Hou, Z., Niyaz, M., Turghun, A., Hasim, A., et al. (2018). High MLL2 Expression Predicts Poor Prognosis and Promotes Tumor Progression by Inducing EMT in Esophageal Squamous Cell Carcinoma. J. Cancer Res. Clin. Oncol. 144, 1025-1035. doi:10.1007/s00432-018-2625-5

Aithal, A., Rauth, S., Kshirsagar, P., Shah, A., Lakshmanan, I., Junker, W. M., et al. (2018). MUC16 as a Novel Target for Cancer Therapy. Expert Opin. Ther. Targets 22, 675-686. doi:10.1080/14728222.2018.1498845

Alam, H., Tang, M., Maitituoheti, M., Dhar, S. S., Kumar, M., Han, C. Y., et al. (2020). KMT2D Deficiency Impairs Super-enhancers to Confer a Glycolytic Vulnerability in Lung Cancer. Cancer Cell 37, 599-617. doi:10.1016/ j.ccell.2020.03.005

Alborelli, I., Leonards, K., Rothschild, S. I., Leuenberger, L. P., and Jermann, P. (2020). Tumor Mutational burden Assessed by Targeted NGS Predicts Clinical Benefit from Immune Checkpoint Inhibitors in Non-small Cell Lung Cancer. J. Pathol., 25019-25029. doi:10.1002/path.5344

Alexandrov, L. B., Kim, J., Kim, J., Haradhvala, N. J., Huang, M. N., Tian Ng, A. W., et al. (2020). The Repertoire of Mutational Signatures in Human Cancer. Nature 578, 94-101. doi:10.1038/s41586-020-1943-3

Arnedo-Pac, C., Mularoni, L., Muiños, F., Gonzalez-Perez, A., and Lopez-Bigas, N. (2019). OncodriveCLUSTL: a Sequence-Based Clustering Method to Identify Cancer Drivers. Bioinformatics 35, 4788-4790. doi:10.1093/bioinformatics/btz501
Our results can deepen the understanding of the mutations of ESCC. Based on the mutation data of large samples, we can identify potential biomarkers of ESCC, especially markers for immunotherapy, and provide new ideas for the diagnosis and treatment of ESCC.

\section{DATA AVAILABILITY STATEMENT}

The original contributions presented in the study are included in the article/Supplementary Material, further inquiries can be directed to the corresponding authors.

\section{AUTHOR CONTRIBUTIONS}

BZ performed the study, analyzed the omics and experimental data and wrote the manuscript. DG performed the experiment. QW and PK revised the manuscript. XC and YC designed the study and revised the manuscript.

\section{FUNDING}

This work was supported by the National Natural Science Foundation of China (81972613, 81672768), the Fund for Shanxi "1331 Project", and the funding for "Sanjin Scholars".

\section{SUPPLEMENTARY MATERIAL}

The Supplementary Material for this article can be found online at: https://www.frontiersin.org/articles/10.3389/fmolb.2021.792779/ full\#supplementary-material

Blass, E., and Ott, P. A. (2021). Advances in the Development of Personalized Neoantigen-Based Therapeutic Cancer Vaccines. Nat. Rev. Clin. Oncol. 18, 215-229. doi:10.1038/s41571-020-00460-2

Boumber, Y. (2018). Tumor Mutational burden (TMB) as a Biomarker of Response to Immunotherapy in Small Cell Lung Cancer. J. Thorac. Dis. 10, 4689-4693. doi:10.21037/jtd.2018.07.120

Boumber, Y. (2018). Tumor Mutational burden (TMB) as a Biomarker of Response to Immunotherapy in Small Cell Lung Cancer. J. Thorac. Dis. 10, 4689. doi: $10.21037 /$ jtd.2018.07.120

Cao, W., Wu, W., Yan, M., Tian, F., Ma, C., Zhang, Q., et al. (2015). Multiple Region Whole-Exome Sequencing Reveals Dramatically Evolving Intratumor Genomic Heterogeneity in Esophageal Squamous Cell Carcinoma. Oncogenesis 4, e175. doi:10.1038/oncsis.2015.34

Chaffer, C. L., and Weinberg, R. A. (2011). A Perspective on Cancer Cell Metastasis. Science 331, 1559-1564. doi:10.1126/science.1203543

Chakravarty, D., Gao, J., Phillips, S., Kundra, R., Zhang, H., Wang, J., et al. (2017). OncoKB: A Precision Oncology Knowledge Base. JCO Precision Oncol. 1, 1-16. doi:10.1200/po.17.00011

Chan, T. A., Yarchoan, M., Jaffee, E., Swanton, C., Quezada, S. A., Stenzinger, A., et al. (2019). Development of Tumor Mutation burden as an Immunotherapy Biomarker: Utility for the Oncology Clinic. Ann. Oncol. 30, 44-56. doi:10.1093/ annonc/mdy495

Chang, J., Tan, W., Ling, Z., Xi, R., Shao, M., Chen, M., et al. (2017). Genomic Analysis of Oesophageal Squamous-Cell Carcinoma Identifies Alcohol 
Drinking-Related Mutation Signature and Genomic Alterations. Nat. Commun. 8, 15290. doi:10.1038/ncomms 15290

Chen, W., Zheng, R., Baade, P. D., Zhang, S., Zeng, H., Bray, F., et al. (2016). Cancer Statistics in China, 2015. CA: A Cancer J. Clinicians 66, 115-132. doi:10.3322/ caac. 21338

Chen, X.-X., Zhong, Q., Liu, Y., Yan, S.-M., Chen, Z.-H., Jin, S.-Z., et al. (2017). Genomic Comparison of Esophageal Squamous Cell Carcinoma and its Precursor Lesions by Multi-Region Whole-Exome Sequencing. Nat. Commun. 8, 524. doi:10.1038/s41467-017-00650-0

Coulie, P. G., Van den Eynde, B. J., Van Der Bruggen, P., and Boon, T. (2014). Tumour Antigens Recognized by T Lymphocytes: at the Core of Cancer Immunotherapy. Nat. Rev. Cancer 14, 135-146. doi:10.1038/nrc3670

Cui, Y., Chen, H., Xi, R., Cui, H., Zhao, Y., Xu, E., et al. (2020). Whole-genome Sequencing of 508 Patients Identifies Key Molecular Features Associated with Poor Prognosis in Esophageal Squamous Cell Carcinoma. Cell. Res. 30, 902-913. doi:10.1038/s41422-020-0333-6

Dai, J. Y., Wang, X., Buas, M. F., Zhang, C., Ma, J., Wei, B., et al. (2018). Wholegenome Sequencing of Esophageal Adenocarcinoma in Chinese Patients Reveals Distinct Mutational Signatures and Genomic Alterations. Commun. Biol. 1, 174. doi:10.1038/s42003-018-0182-8

Dai, W., Ko, J. M. Y., Choi, S. S. A., Yu, Z., Ning, L., Zheng, H., et al. (2017). Wholeexome Sequencing Reveals Critical Genes Underlying Metastasis in Oesophageal Squamous Cell Carcinoma. J. Pathol. 242, 500-510. doi:10.1002/path.4925

Du, P., Huang, P., Huang, X., Li, X., Feng, Z., Li, F., et al. (2017). Comprehensive Genomic Analysis of Oesophageal Squamous Cell Carcinoma Reveals Clinical Relevance. Sci. Rep. 7, 15324. doi:10.1038/s41598-017-14909-5

Dutta, M., Nakagawa, H., Kato, H., Maejima, K., Sasagawa, S., Nakano, K., et al. (2020). Whole Genome Sequencing Analysis Identifies Recurrent Structural Alterations in Esophageal Squamous Cell Carcinoma. PeerJ 8, e9294. doi:10.7717/peer. 9294

Felder, M., Kapur, A., Gonzalez-Bosquet, J., Horibata, S., Heintz, J., Albrecht, R., et al. (2014). MUC16 (CA125): Tumor Biomarker to Cancer Therapy, a Work in Progress. Mol. Cancer 13, 1-15. doi:10.1186/1476-4598-13-129

Felder, M., Kapur, A., Gonzalez-Bosquet, J., Horibata, S., and Patankar, M. S. (2014). MUC16 (CA125): Tumor Biomarker to Cancer Therapy, a Work in Progress. Mol. Cancer 13, 129. doi:10.1186/1476-4598-13-129

Gambacorti-Passerini, C., Orlov, S., Zhang, L., Braiteh, F., Huang, H., Esaki, T., et al. (2018). Long-term Effects of Crizotinib in ALK-positive Tumors (Excluding NSCLC). A phase 16 open-label study 93, 607-614.

Greillier, L., Tomasini, P., and Barlesi, F. (2018). The Clinical Utility of Tumor Mutational burden in Non-small Cell Lung Cancer. Transl. Lung Cancer Res. 7, 639-646. doi:10.21037/tlcr.2018.10.08

Griffith, M., Spies, N. C., Krysiak, K., McMichael, J. F., Coffman, A. C., Danos, A. M., et al. (2017). CIViC Is a Community Knowledgebase for Expert Crowdsourcing the Clinical Interpretation of Variants in Cancer. Nat. Genet. 49, 170-174. doi:10.1038/ng.3774

Guo, C., Chen, L. H., Huang, Y., Chang, C.-C., Wang, P., Pirozzi, C. J., et al. (2013). KMT2D Maintains Neoplastic Cell Proliferation and Global Histone H3 Lysine 4 Monomethylation. Oncotarget 4, 2144. doi:10.18632/oncotarget.1555

Han, Y., Yang, J., Qian, X., Cheng, W.-C., Liu, S.-H., Hua, X., et al. (2019). DriverML: a Machine Learning Algorithm for Identifying Driver Genes in Cancer Sequencing Studies. Nucleic Acids Res. 47, e45. doi:10.1093/nar/gkz096

He, W., Leng, X., Yang, Y., Peng, L., Shao, Y., Li, X., et al. (2020). Genetic Heterogeneity of Esophageal Squamous Cell Carcinoma with Inherited Family History. Ott Vol. 13, 8795-8802. doi:10.2147/ott.s262512

Hu, N., Kadota, M., Liu, H., Abnet, C. C., Su, H., Wu, H., et al. (2016). Genomic Landscape of Somatic Alterations in Esophageal Squamous Cell Carcinoma and Gastric Cancer. Cancer Res. 76, 1714-1723. doi:10.1158/0008-5472.can-150338

Hundal, J., Kiwala, S., McMichael, J., Miller, C. A., Xia, H., Wollam, A. T., et al. (2020). pVACtools: a Computational Toolkit to Identify and Visualize Cancer Neoantigens. Cancer Immunol. Res. 8, 409-420. doi:10.1158/2326-6066.CIR19-0401

Jardim, D. L., Goodman, A., Gagliato, D., and Kurzrock, R. (2020). The Challenges of Tumor Mutational Burden as an Immunotherapy Biomarker. Cancer Cell 39, 154-173. doi:10.1016/j.ccell.2020.10.001
Jou, J., Harrington, K. J., Zocca, M.-B., Ehrnrooth, E., and Cohen, E. (2021). The Changing Landscape of Therapeutic Cancer Vaccines-Novel Platforms and Neoantigen Identification. Clin. Cancer Res. 27, 689-703. doi:10.1158/10780432.ccr-20-0245

Kassambara, A., Kosinski, M., Biecek, P., and Fabian, S. (2017). Survminer: Drawing Survival Curves Usin gggplot2', R package version 0.3, 1.

Koshy, M., Esiashvilli, N., Landry, J. C., Thomas, C. R., and Matthews, R. H. (2004). Multiple Management Modalities in Esophageal Cancer: Combined Modality Management Approaches. The Oncologist 9, 147-159. doi:10.1634/ theoncologist.9-2-147

Lai, R., Zhang, W., He, X., Liao, X., Liu, X., Fu, W., et al. (2020). Prognostic Role of ACTL10 in Cytogenetic Normal Acute Myeloid Leukemia. J. Cancer 11, 5150. doi:10.7150/jca.39467

Lawrence, M. S., Stojanov, P., Polak, P., Kryukov, G. V., Cibulskis, K., Sivachenko, A., et al. (2013). Mutational Heterogeneity in Cancer and the Search for New Cancer-Associated Genes. Nature 499, 214-218. doi:10.1038/nature12213

Li, S.-S., Jiang, W.-L., Xiao, W.-Q., Li, K., Zhang, Y.-F., Guo, X.-Y., et al. (2019). KMT2D Deficiency Enhances the Anti-cancer Activity of L48H37 in Pancreatic Ductal Adenocarcinoma. World J. Gastrointest. Oncol. 11, 599. doi:10.4251/ wjgo.v11.i8.599

Lin, D.-C., Hao, J.-J., Nagata, Y., Xu, L., Shang, L., Meng, X., et al. (2014). Genomic and Molecular Characterization of Esophageal Squamous Cell Carcinoma. Nat. Genet. 46, 467-473. doi:10.1038/ng.2935

Liu, M., An, H., Zhang, Y., Sun, W., Cheng, S., Wang, R., et al. (2020). Molecular Analysis of Chinese Oesophageal Squamous Cell Carcinoma Identifies Novel Subtypes Associated with Distinct Clinical Outcomes. EBioMedicine 57, 102831. doi:10.1016/j.ebiom.2020.102831

Liu, X., Zhang, M., Ying, S., Zhang, C., Lin, R., Zheng, J., et al. (2017). Genetic Alterations in Esophageal Tissues from Squamous Dysplasia to Carcinoma. Gastroenterology 153, 166-177. doi:10.1053/j.gastro.2017.03.033

Lu, Y.-C., and Robbins, P. F. (2016). Cancer Immunotherapy Targeting Neoantigens, Seminars in Immunology. Amsterdam, Netherlands: Elsevier, 22-27. doi:10.1016/j.smim.2015.11.002

Łuksza, M., Riaz, N., Makarov, V., Balachandran, V. P., Hellmann, M. D., Solovyov, A., et al. (2017). A Neoantigen Fitness Model Predicts Tumour Response to Checkpoint Blockade Immunotherapy. Nature 551, 517-520.

Mangalaparthi, K. K., Patel, K., Khan, A. A., Manoharan, M., Karunakaran, C., Murugan, S., et al. (2020). Mutational Landscape of Esophageal Squamous Cell Carcinoma in an Indian Cohort. Front. Oncol. 10, 1457. doi:10.3389/ fonc. 2020.01457

Martincorena, I., Raine, K. M., Gerstung, M., Dawson, K. J., Haase, K., Van Loo, P., et al. (2017). Universal Patterns of Selection in Cancer and Somatic Tissues. Cell 171, 1029-1041. doi:10.1016/j.cell.2017.09.042

Martínez-Jiménez, F., Muiños, F., Sentís, I., Deu-Pons, J., Reyes-Salazar, I., ArnedoPac, C., et al. (2020). A Compendium of Mutational Cancer Driver Genes. Nat. Rev. Cancer 20, 555-572. doi:10.1038/s41568-020-0290-x

Mayakonda, A., Lin, D.-C., Assenov, Y., Plass, C., and Koeffler, H. P. (2018). Maftools: Efficient and Comprehensive Analysis of Somatic Variants in Cancer. Genome Res. 28, 1747-1756. doi:10.1101/gr.239244.118

McLaren, W., Gil, L., Hunt, S. E., Riat, H. S., Ritchie, G. R., Thormann, A., et al. (2016). The ensembl variant effect predictor 17, 1-14. doi:10.1186/s13059-016-0974-4

Mularoni, L., Sabarinathan, R., Deu-Pons, J., Gonzalez-Perez, A., and López-Bigas, N. (2016). OncodriveFML: a General Framework to Identify Coding and NonCoding Regions with Cancer Driver Mutations. Genome Biol. 17, 128. doi:10.1186/s13059-016-0994-0

Nature, C. (2017). Integrated Genomic Characterization of Oesophageal Carcinoma. Nature 541, 169-175. doi:10.1038/nature20805

Nie, W., Xu, M., Gan, L., Zhang, Y., and Han, B. H. (2020). Advanced Non-small Cell Lung Cancer Patients with Low Tumor Mutation Burden Might Derive Benefit from Immunotherapy. J. Immunother. 43, 189-195. doi:10.1097/ CJI.0000000000000318

Qin, H.-D., Liao, X.-Y., Chen, Y.-B., Huang, S.-Y., Xue, W.-Q., Li, F.-F., et al. (2016). Genomic Characterization of Esophageal Squamous Cell Carcinoma Reveals Critical Genes Underlying Tumorigenesis and Poor Prognosis. Am. J. Hum. Genet. 98, 709-727. doi:10.1016/j.ajhg.2016.02.021

Samstein, R. M., Lee, C.-H., Shoushtari, A. N., Hellmann, M. D., Shen, R., Janjigian, Y. Y., et al. (2019). Tumor Mutational Load Predicts Survival after 
Immunotherapy across Multiple Cancer Types. Nat. Genet. 51, 202-206. doi:10.1038/s41588-018-0312-8

Sawada, G., Niida, A., Uchi, R., Hirata, H., Shimamura, T., Suzuki, Y., et al. (2016). Genomic Landscape of Esophageal Squamous Cell Carcinoma in a Japanese Population. Gastroenterology 150, 1171-1182. doi:10.1053/j.gastro.2016.01.035

Schumacher, T. N., and Schreiber, R. (2015). Neoantigens in Cancer Immunotherapy. Science 348, 69-74. doi:10.1126/science.aaa4971

Snyder, A., and Chan, T. A. (2015). Immunogenic Peptide Discovery in Cancer Genomes. Curr. Opin. Genet. Dev. 30, 7-16. doi:10.1016/j.gde.2014.12.003

Song, Y., Li, L., Ou, Y., Gao, Z., Li, E., Li, X., et al. (2014). Identification of Genomic Alterations in Oesophageal Squamous Cell Cancer. Nature 509, 91-95. doi:10.1038/nature13176

Subbiah, V., Solit, D. B., Chan, T. A., and Kurzrock, R. (2020). The FDA Approval of Pembrolizumab for Adult and Pediatric Patients with Tumor Mutational burden $(\mathrm{TMB}) \geq 10$ : a Decision Centered on Empowering Patients and Their Physicians. Ann. Oncol. 31, 1115-1118. doi:10.1016/ j.annonc.2020.07.002

Sun, P., Wu, T., Sun, X., Cui, Z., Zhang, H., Xia, Q., et al. (2019). KMT2D Inhibits the Growth and Metastasis of Bladder Cancer Cells by Maintaining the Tumor Suppressor Genes. Biomed. Pharmacother. 115, 108924. doi:10.1016/ j.biopha.2019.108924

Toska, E., Osmanbeyoglu, H. U., Castel, P., Chan, C., Hendrickson, R. C., Elkabets, M., et al. (2017). PI3K Pathway Regulates ER-dependent Transcription in Breast Cancer through the Epigenetic Regulator KMT2D. Science 355, 1324-1330. doi:10.1126/science.aah6893

Türeci, Ö., Löwer, M., Schrörs, B., Lang, M., Tadmor, A., and Sahin, U. (2018). Challenges towards the Realization of Individualized Cancer Vaccines. Nat. Biomed. Eng. 2, 566-569. doi:10.1038/s41551-018-0266-2

Wang, S., Tao, Z., Wu, T., and Liu, X.-S. (2020). Sigflow: an Automated and Comprehensive Pipeline for Cancer Genome Mutational Signature Analysis. Bioinformatics. doi:10.1093/bioinformatics/btaa895

Wang, Z., Pan, L., Guo, D., Luo, X., Tang, J., Yang, W., et al. (2020). A Novel FiveGene Signature Predicts Overall Survival in Hepatocellular Carcinoma. Front. Oncol. doi:10.3389/fonc.2021.642563

Wu, Z., Long, X., Tsang, S. Y., Hu, T., Yang, J.-F., Mat, W. K., et al. (2020). Genomic Subtyping of Liver Cancers with Prognostic Application. BMC Cancer 20, 1-13. doi:10.1186/s12885-020-6546-8

Xie, C., Mao, X., Huang, J., Ding, Y., Wu, J., Dong, S., et al. (2011). KOBAS 2.0: a Web Server for Annotation and Identification of Enriched Pathways and Diseases. Nucleic Acids Res. 39, W316-W322. doi:10.1093/nar/gkr483

Xiong, W., Deng, Z., Tang, Y., Deng, Z., Li, M. J. B., and communications, b. r. (2018). Downregulation of KMT2D Suppresses Proliferation and Induces Apoptosis of Gastric Cancer. Biochem. Biophys. Res. Commun. 504, 129-136. doi:10.1016/j.bbrc.2018.08.143

Xu, Z., Dai, J., Wang, D., Lu, H., Dai, H., Ye, H., et al. (2019). Assessment of Tumor Mutation burden Calculation from Gene Panel Sequencing Data. Ott Vol. 12, 3401-3409. doi:10.2147/ott.s196638

Yan, T., Cui, H., Zhou, Y., Yang, B., Kong, P., Zhang, Y., et al. (2019). Multi-region Sequencing Unveils Novel Actionable Targets and Spatial Heterogeneity in Esophageal Squamous Cell Carcinoma. Nat. Commun. 10, 1670. doi:10.1038/ s41467-019-09255-1

Yeku, O. O., Rafiq, S., Koneru, M., Purdon, T. J., Song, M., Wang, P., et al. (2020). MUC16-directed Immunotherapy for Ovarian Cancer. Philadelphia, PA:
Clinical Cancer Research, Amer Assoc Cancer Research 615 Chestnut ST, 17th Floor, 26.

Yin, L., Zhou, L., and Xu, R. (2021). Identification of Tumor Mutation burden and Immune Infiltrates in Hepatocellular Carcinoma Based on Multi-Omics Analysis. Front. Mol. Biosci. 7, 496. doi:10.3389/fmolb.2020.599142

Younes, M., Henson, D. E., Ertan, A., and Miller, C. C. (2002). Incidence and Survival Trends of Esophageal Carcinoma in the United States: Racial and Gender Differences by Histological Type. Scand. J. Gastroenterol. 37, 1359-1365. doi:10.1080/003655202762671215

Yu, Y., Cao, J., Wu, W., Zhu, Q., Tang, Y., Zhu, C., et al. (2019). Genome-wide Copy Number Variation Analysis Identified ANO1 as a Novel Oncogene and Prognostic Biomarker in Esophageal Squamous Cell Cancer. Carcinogenesis 40, 1198-1208. doi:10.1093/carcin/bgz077

Yu, Y., Lin, D., Li, A., Chen, Y., and Yao, H. (2020). Association of Immune Checkpoint Inhibitor Therapy with Survival in Patients with Cancers with MUC16 Variants. JAMA Netw. Open 3, e205837. doi:10.1001/ jamanetworkopen.2020.5837

Zehir, A., Benayed, R., Shah, R. H., Syed, A., Middha, S., Kim, H. R., et al. (2017). Mutational Landscape of Metastatic Cancer Revealed from Prospective Clinical Sequencing of 10,000 Patients. Nat. Med. 23, 703-713. doi:10.1038/nm.4333

Zhang, L., Zhou, Y., Cheng, C., Cui, H., Cheng, L., Kong, P., et al. (2015). Genomic Analyses Reveal Mutational Signatures and Frequently Altered Genes in Esophageal Squamous Cell Carcinoma. Am. J. Hum. Genet. 96, 597-611. doi:10.1016/j.ajhg.2015.02.017

Zheng, B., Song, Z., Chen, Y., and Yan, W. (2021). Genomic Analyses of Metaplastic or Sarcomatoid Carcinomas from Different Organs Revealed Frequent Mutations in KMT2D. Front. Mol. Biosci. 8, 587. doi:10.3389/ fmolb.2021.688692

Zhou, C., Li, C., Peng, S., Zhou, L., and Li, H. (2020). C, 7. Omprehensive Analysis of the Relationships between Tumor Mutation burden with Immune Infiltrates in Cervical Cell Carcinoma. Front. Mol. Biosci. doi:10.3389/fmolb.2020.582911

Zhou, R., Zeng, D., Zhang, J., Sun, H., Wu, J., Li, N., et al. (2019). A Robust Panel Based on Tumour Microenvironment Genes for Prognostic Prediction and Tailoring Therapies in Stage I-III colon Cancer. EBioMedicine 42, 420-430. doi:10.1016/j.ebiom.2019.03.043

Conflict of Interest: The authors declare that the research was conducted in the absence of any commercial or financial relationships that could be construed as a potential conflict of interest.

Publisher's Note: All claims expressed in this article are solely those of the authors and do not necessarily represent those of their affiliated organizations, or those of the publisher, the editors and the reviewers. Any product that may be evaluated in this article, or claim that may be made by its manufacturer, is not guaranteed or endorsed by the publisher.

Copyright $\odot 2022$ Zou, Guo, Kong, Wang, Cheng and Cui. This is an open-access article distributed under the terms of the Creative Commons Attribution License (CC BY). The use, distribution or reproduction in other forums is permitted, provided the original author(s) and the copyright owner(s) are credited and that the original publication in this journal is cited, in accordance with accepted academic practice. No use, distribution or reproduction is permitted which does not comply with these terms. 